\title{
Voltage-sensitive dye imaging reveals inhibitory modulation of ongoing cortical activity
}

\author{
Taylor H. Newton ${ }^{1, *}$, Marwan Abdellah ${ }^{1}$, Grigori Chevtchenko ${ }^{1}$, Eilif B. Muller ${ }^{1}$, and Henry \\ Markram ${ }^{1,2}$ \\ ${ }^{1}$ Blue Brain Project, École polytechnique fédérale de Lausanne (EPFL), Campus Biotech, 1202 \\ Geneva, Switzerland \\ ${ }^{2}$ Laboratory of Neural Microcircuitry, Brain Mind Institute, EPFL, 1015 Lausanne, Switzerland \\ *Correspondence: taylor.newton@epfl.ch
}

\begin{abstract}
Voltage-sensitive dye imaging (VSDI) is a powerful technique for interrogating membrane potential dynamics in assemblies of cortical neurons, but with effective resolution limits that confound interpretation. In particular, it is unclear how VSDI signals relate to population firing rates. To address this limitation, we developed an in silico model of VSDI in a biologically faithful digital reconstruction of rodent neocortical microcircuitry. Using this model, we extend previous experimental observations regarding the cellular origins of VSDI, finding that the signal is driven primarily by neurons in layers $2 / 3$ and 5 . We proceed by exploring experimentally inaccessible circuit properties to show that during periods of spontaneous activity, membrane potential fluctuations are anticorrelated with population firing rates. Furthermore, we manipulate network connections to show that this effect depends on recurrent connectivity and is modulated by external input. We conclude that VSDI primarily reflects inhibitory responses to ongoing excitatory dynamics.
\end{abstract}

Keywords: in silico modeling, voltage-sensitive dyes, mesoscale imaging, spatiotemporal dynamics, feedback inhibition

\section{Main}

Electrical signaling in cortex is thought to be divided into "inputs" in the form of subthreshold synaptic potentials, and "outputs" in the form of action potentials (APs) or spikes (Grinvald and Hildesheim, 2004). Therefore, a complete understanding of cortical function requires not only a means of recording spikes in neural ensembles, but also a technique for resolving subthreshold membrane potentials $\left(V_{m}\right)$ in these populations. Voltage-sensitive dye imaging (VSDI) is a mesoscale imaging technique capable 
of capturing subthreshold activity across the entire rodent neocortical surface (on the order of several $\mathrm{cm}^{2}$ ) with good spatiotemporal resolution (on the order of milliseconds in time, and $<50 \mu \mathrm{m}$ in space) (Chemla and Chavane, 2010; Ferezou et al., 2009; Grinvald and Hildesheim, 2004; Shoham et al., 1999).

In previous decades, significant progress was made in mapping the functional architecture of the brain using intrinsic optical imaging, a modality based on activity-dependent changes in the intrinsic absorptive and fluorescent properties of brain tissue. Studies combining intrinsic optical imaging with cytochrome oxidase staining revealed the interdependent organization of ocular dominance columns, cytochrome oxidase blobs (color preference), and orientation selective "pinwheel" structures in the visual system (Bartfeld and Grinvald, 1992; Blasdel, 1992a, 1992b; Frostig et al., 1990; Ts'o et al., 1990). However, intrinsic optical imaging is limited by a slow time constant (on the order of seconds (Grinvald et al., 1986, 2016)), rendering it ill-suited for capturing temporal changes in ongoing activity. To this end, VSDI has added a dynamic component to the understanding of neural assemblies. For example, VSDI-based studies have clarified the role of feedforward thalamic inputs versus intracortical recurrent activity in shaping orientation selectivity (Sharon and Grinvald, 2002), and shown how orientation-selective responses spread over the cortex as a function of stimulus shape and size (Chavane et al., 2011). VSDI has also been widely applied to the study of somatosensory computations in barrel cortex, where the somatotopic organization and spatiotemporal scale of activity is well suited to the technique. Such studies have produced important findings regarding the regulation of response dynamics by ongoing spontaneous activity (Petersen et al., 2003a), cortical state (Civillico and Contreras, 2012), behavior (Ferezou et al., 2006, 2007; Kyriakatos et al., 2017), and stimulus strength (Petersen et al., 2003b). Broadly put, VSDI has enabled the field to move beyond the static picture provided by staining and intrinsic optical imaging, adding a dynamic dimension to the understanding of mesoscale cortical organization.

However, VSDI suffers from the limitation that the superposed activity of neurites belonging to many cells is reflected in each image pixel. Uneven dye penetration, and blurring due to absorption and scattering of photons in tissue further complicate the interpretation of VSDI signals (Chemla and Chavane, 2010; Grinvald and Hildesheim, 2004; Grinvald et al., 2015). Indeed, a historical concern has been identifying which attributes of neural anatomy and physiology (e.g. layer, cell type, dendrites vs. axons, pre- vs. postsynaptic activity) are the primary drivers of VSDI measurements (Civillico and Contreras, 2006; Ferezou et al., 2006; Lippert et al., 2007; Petersen et al., 2003b). A model of VSDI 
that considers both the biological organization of the neuronal tissue and the physics of signal acquisition could answer these questions. However, the true power of such a model would lie not merely in inferring the relative importance of different aspects of neural activity, but also in bridging spatiotemporal scales to generate new insights into the emergent dynamics of cortical populations.

Here, we present the results of a detailed computational model of VSDI, implemented in a digital reconstruction of rodent neocortical microcircuitry (NMC), specifically, the hindlimb somatosensory cortex of a juvenile rat (Markram et al., 2015). The NMC comprises a network of $\sim 31,000$ neurons with detailed cellular anatomy and physiology and data-driven synaptic physiology, arranged with algorithmically constrained connectivity in a $0.29 \pm 0.01 \mathrm{~mm}^{3}$ column of tissue (Fig. 1a,b). To simulate VSDI signals, we performed simulations of the NMC to obtain $V_{m}$ recordings of neural compartments under various experimental conditions (Fig. 1c) (see also Supplementary Fig. 2 for an evaluation of evoked VSDI in individual statistical instantiations of the NMC). Next, we corrected the compartment voltages to account for the effects of dye penetration and light transport in tissue (Fig. 1d), and collected this data into voxels (Fig. 1d,e). Using offline Monte Carlo simulations of photon-tissue interactions and a ray transfer model of microscope optics, we calculated a depth-dependent point spread function (PSF), with which we convolved horizontal slices of voxelized data (Fig. 1e,f) (see also Methods). This procedure generated a time-ordered collection of VSD images (Fig. 1g), which we repeated for various permutations of microcircuit geometry (Fig. $1 \mathrm{~h}$ ) to probe population dynamics in the NMC model.

\section{Results}

\section{Evoked VSDI response dynamics}

Propagating waves of activity support the representation and integration of information in cortex (Borgdorff et al., 2007; Contreras and Llinás, 2001; Ferezou et al., 2006, 2007; Lustig et al., 2013; Petersen and Sakmann, 2001; Petersen et al., 2003b), and can be observed with VSDI. Activity spread dynamics are commonly characterized by measuring the phase velocity and aspect ratio of the propagating wavefront, the spatial extent and time-course of cortical activation, and the dependence of these quantities on stimulus strength (cf. Fehérvári et al., 2015). To quantify the similarity between the evoked response dynamics of our model and those reported in literature, we conducted a series of whisker flick-like trials and examined the spread of activity. Our stimulation protocol consisted of a single pulse of activity in 60 contiguous thalamocortical fibers emanating from a virtual ventral 


\section{Fig. 1: Cortical microcircuit overview and in silico VSDI workflow}

$a$

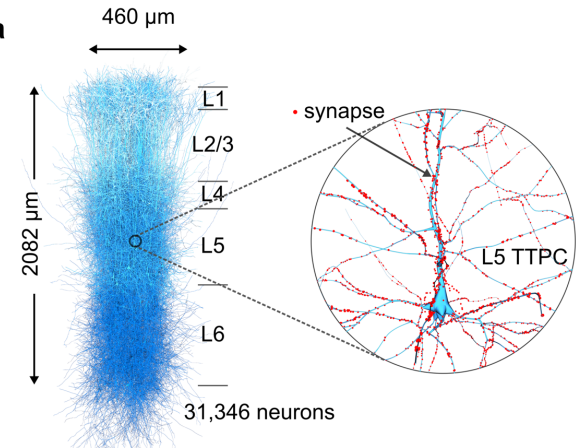

C
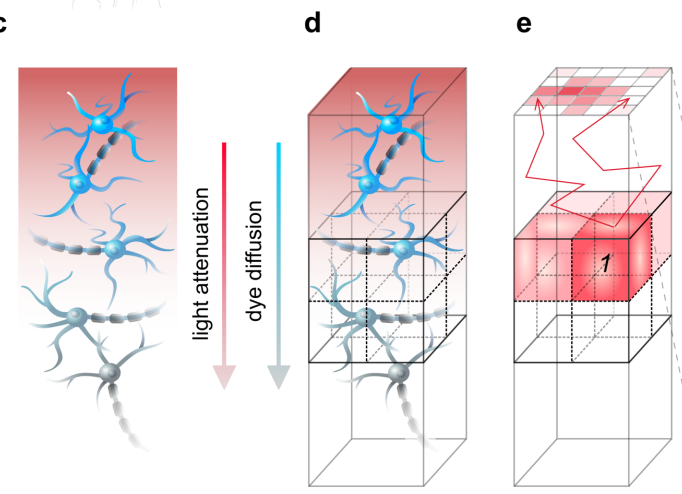

b

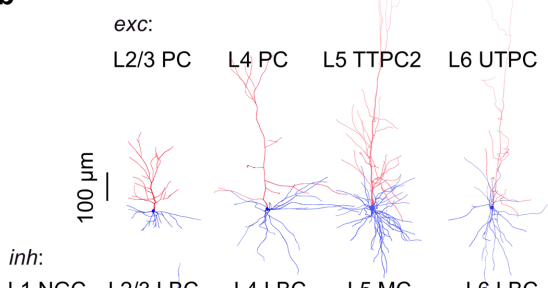

L1 NGC L2/3 LBC L4 LBC L5 MC L6 LBC

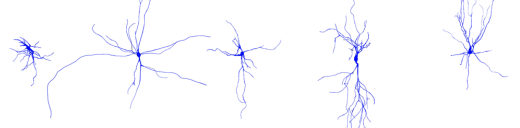

g

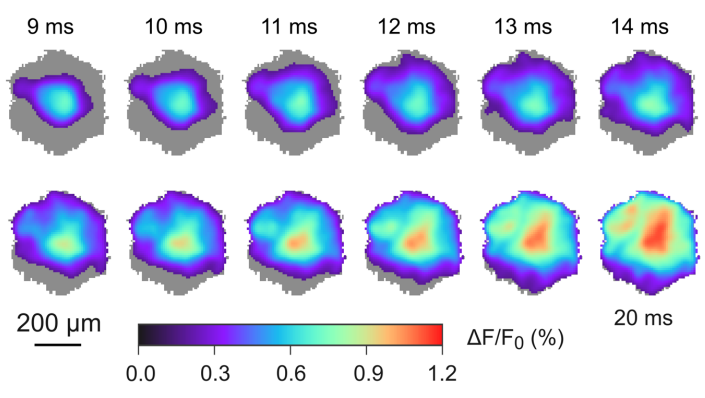

f

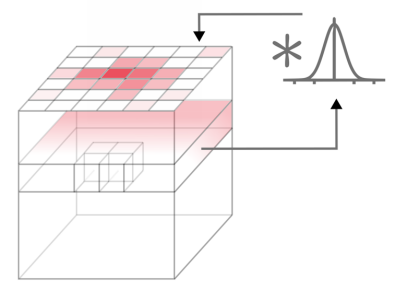

h

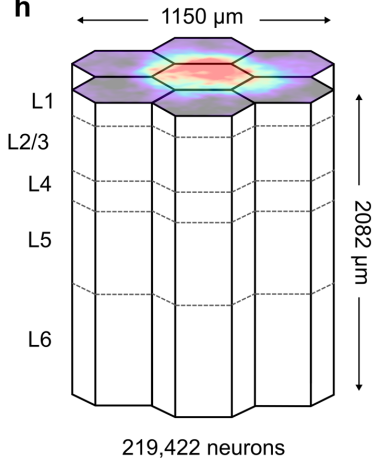

a, Digital microcircuit comprising 31,346 morphologically detailed neurons connected in a columnar unit. Inset: expanded view of a L5 PC, with synapses highlighted in red. b, Exemplar excitatory (top) and inhibitory (bottom) cell types. Blue: axons. Red: dendrites. c-f, Schematic illustrating the in silico VSDI workflow. c, Neuron surface areas are scaled by prefactors accounting for dye diffusion and light transport in cortical tissue. d, Microcircuit volume is divided into voxels to facilitate calculations. e, Photons emitted from each voxel are scattered and absorbed throughout the tissue volume via Monte Carlo simulations. Photons reaching the cortical surface are propagated through a tandemlens optical setup using ray transfer matrix analysis. Steps $\mathbf{e}$ and $\mathbf{f}$ are performed once for a given circuit and optical setup to determine a depth-dependent point-spread function (point-spread from 1 to 2 in panel, i.e. from voxel to camera). f, Raw signals at each depth are convolved with their respective point-spread function, and accumulated in a pixel array at the surface. g, Example VSDI image stack for $11 \mathrm{~ms}$ of spontaneous activity. Images were thresholded at $10 \%$ of peak response. $\mathbf{h}$, Microcircuits were aggregated into a larger volume made of a central microcircuit column surrounding by six additional columns contacting each of the central column's hexagonal sides (the "mosaic"). This arrangement mitigates boundary effects within the central column and facilitates the analysis of signal spread dynamics. 
posteromedial nucleus (VPM) projecting to the geometric center of a concentric arrangement of 7 NMCs (the "mosaic", Fig. 1h). We observed a radially expanding pattern of activation centered around the location of stimulus delivery, which expanded to fill the entire NMC surface over the course of several tens of milliseconds, reaching peak fluorescence at $\sim 57 \mathrm{~ms}$ poststimulus (Fig. 2a,b). The peak was immediately followed by a period of declining activity characterized by increasing hyperpolarization, which undershot baseline fluorescence, reaching a minimum at $170 \mathrm{~ms}$ and gradually recovering to within $10 \%$ of baseline after $\sim 510 \mathrm{~ms}$. To quantify the temporal persistence of the signal, we calculated the half width duration (decay time to $50 \%$ of signal peak from baseline, 88 $\mathrm{ms}$ ). The time to peak and half width duration compared favorably to the findings of Ferezou et al., 2006 , who report values of $\sim 45 \mathrm{~ms}$, and $86 \pm 69 \mathrm{~ms}$, respectively (Fig. $2 \mathrm{~b}$ ). We also considered the relationship between the instantaneous firing rate ( $3 \mathrm{~ms}$ bins) and the VSDI signal in a $100 \mathrm{~ms}$ poststimulus window (Fig. 2c,d). Our simulations indicate that peak AP firing occurred $\sim 7$ ms prior to peak VSD fluorescence, contrary to the intuition that increased mean $V_{m}$ precipitates population spiking.

Notably, a differing VSD activity time-course was observed at the stimulus delivery location relative to the NMC periphery (Fig. $2 \mathrm{~g}$ ). At peripheral points along the $x$ - and $z$-axes $(+540 \mu \mathrm{m}$ and $+460 \mu \mathrm{m}$, respectively), the rising and falling phases of the fluorescence response were almost identical to the spatial mean. In contrast, signal recorded at the stimulus location exhibited an initial transient within the first 12 ms of stimulus onset, and then gradually rose to peak fluorescence. This response pattern (initially confined, expanding thereafter) was also visible in the spatial profile of activation over time as an initially sharp peak, which gradually rose and then flattened into a plateau (Fig. 2f).

In order to characterize the propagation velocity of the evoked activity wavefront, we fit each image frame to a two-dimensional Gaussian surface and measured the change in the full width at half maximum (FWHM) over time (Fig. 2e) (see Supplementary Algorithm 1 for details). We found that activity wavefronts underwent two sequential bursts of expansion prior to peak VSD fluorescence, reaching a peak velocity of $\sim 20 \mu \mathrm{m} / \mathrm{ms}$. Subsequently, the wavefront entered a period of contraction ($10 \mu \mathrm{m} / \mathrm{ms}$ ) near the fluorescence peak, before gradually returning to baseline (fluctuations near zero). In vivo VSDI experiments have documented wavefront propagation speeds within an order of magnitude of those reported above. For example, Petersen et al., 2003a use a Gaussian fit of the cross-sectional profile of VSD images to estimate that whisker deflection-evoked waves in urethane or halothane anesthetized rodent barrel cortex propagate along barrel rows at a speed of $\sim 60 \mu \mathrm{m} / \mathrm{ms}$, and barrel arcs at $\sim 33 \mu \mathrm{m} / \mathrm{ms}$. See Supplementary Table 1 for a summary of cortical wavefront propagation 


\section{Fig. 2: Propagation of stimulus-evoked cortical activity}

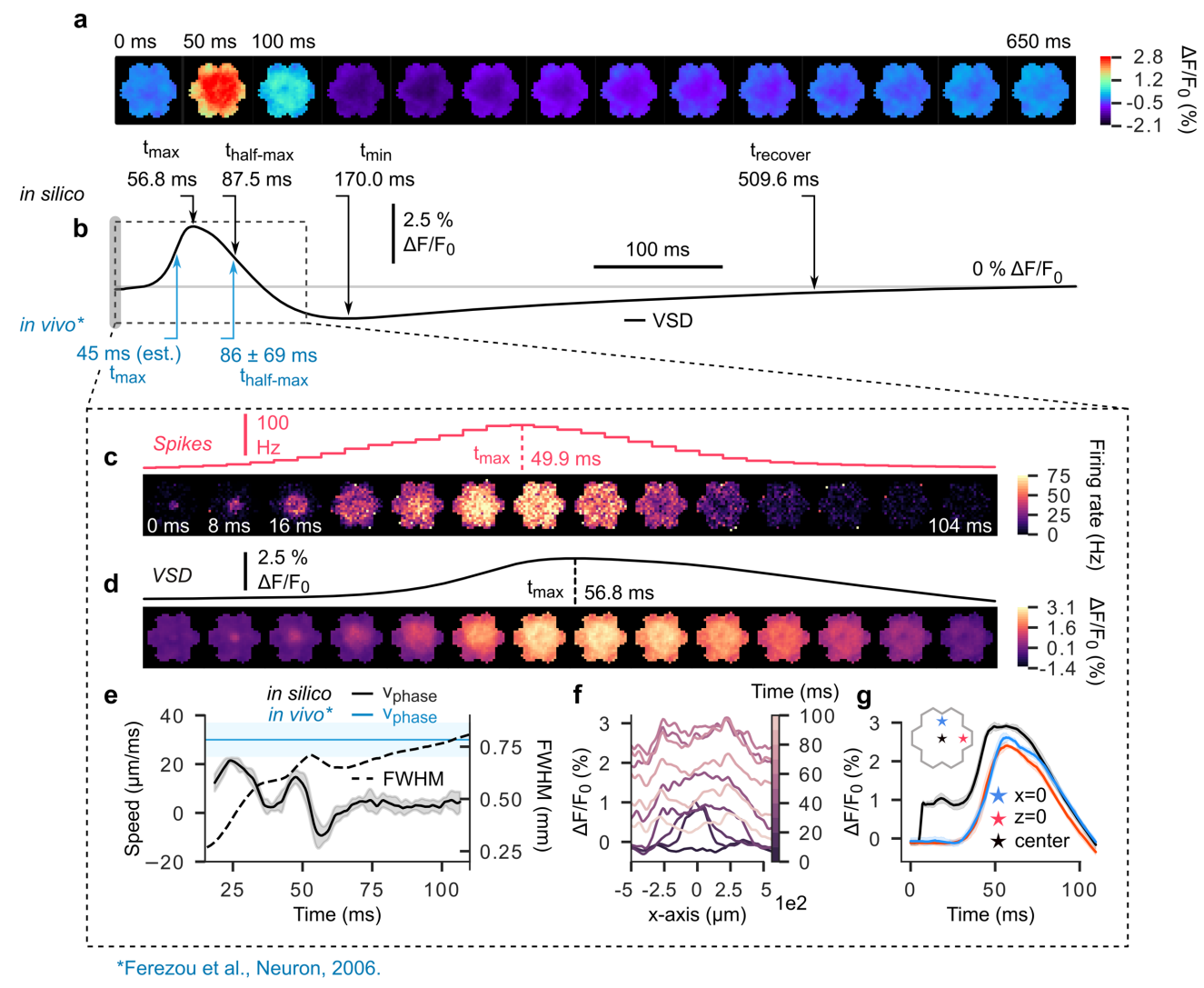

a, 650 milliseconds of simulated stimulus-evoked VSD signals in the mosaic. The stimulus consists of a single, coincident pulse of activity in 60 contiguous thalamic projection fibers innervating the center of the interior microcircuit (delivered at $\mathrm{t}=0 \mathrm{~ms}$ ). $\mathbf{b}$, Time course of spatiallyaveraged VSD signal in a. Upper arrows (black) indicate points of interest along the curve. From left to right: peak latency, half-maximum duration (time to decay to $50 \%$ of signal peak), time of signal minimum, recovery time (earliest time after minimum for amplitude to stably decay to within $10 \%$ of baseline). Bottom arrows (blue) indicate in vivo values for peak latency and half-maximum duration reported in literature. c, Top: PSTH of spiking activity for time window (dashed box) indicated in b (3 ms bins). Bottom: pixel-wise PSTH (mean firing rate of all cells under each pixel) over same time window (8 ms bins). d,Top: expanded view of time window (dashed box) indicated in $\mathbf{b}$, detailing ascending and descending phases of stimulus-evoked spatially-averaged VSD signal. Bottom: same as above, but for spatially extended VSD signal, where each frame was computed by averaging activity in $8 \mathrm{~ms}$ intervals. e, Activity wavefront propagation velocity (solid line, left axis), and wavefront size (full-width at half maximum, dashed line, right axis). Blue horizontal line and shaded region show in vivo measurements of stimulus-evoked wavefront propagation velocity reported in literature. Solid blue line: mean value, shaded blue region: measurement range. $\mathbf{f}$, Spatiotemporal evolution of VSD signal along the $x$-axis $(z=0$, i.e. a horizontal line across the surface of the mosaic). Lightening hue represents passage of time. $\mathbf{g}$, Time course of VSD activation at specific locations on the cortical surface. Blue star: circuit periphery along the $\mathrm{y}$-axis. Red star: circuit periphery along the $\mathrm{x}$-axis. Black star: circuit center.

velocities reported in literature. Also, see Supplementary Fig. 3 for an analysis of laminar VSDI activity spread in a sagittal slice ( $x-y$ plane) of the NMC. 


\section{Excitatory neurons in layers $2 / 3$ and 5 dominate VSDI measurements}

VSDI signals are linearly proportional to the product of local $V_{m}$ and membrane surface area (Grinvald and Hildesheim, 2004). Moreover, signals originating in neurites located in deeper layers are significantly more attenuated than those emanating from superficial layers due to uneven dye penetration and light-tissue interactions (Fig. 3b). It follows that the morphology, location, and orientation of a given cell affect the magnitude of its contribution to the optical response. To better understand these influences, we analyzed the fractional contributions of cortical layers and cell types to the overall VSDI signal. In agreement with previously reported results (Ferezou et al., 2007; Gollnick et al., 2016; Lippert et al., 2007; Petersen et al., 2003a), we found that $>90 \%$ of the raw fluorescence originated within $500 \mu \mathrm{m}$ of the pial surface (Fig. 3b). Furthermore, we saw that neurites belonging to L2/3 and L5 neurons monopolized the "effective surface area", which we define as the quantity that results from multiplying the original surface area of each neurite by a depth-dependent scale factor accounting for dye penetration and light transport; L2/3 and L5 contributed $44.9 \%$ and $43.7 \%$ of the total, respectively. As predicted by the distribution of effective surface area, L2/3 and L5 were the primary drivers of the VSDI signal (47.8\% and $37.6 \%$, respectively, $\mathrm{n}=10$ trials) during spontaneous activity (Fig. 3c,d). Cross-correlation revealed mutual positive correlations between the contributions of each layer and the VSDI total (Fig. 3e). However, during evoked activity, L5 contributed upwards of $67 \%$ of the signal whereas L2/3 neurites constituted 19\% (Fig. 3f,g). Importantly, L5 underwent strong depolarization in the poststimulus window while $L 2 / 3$ tended to hyperpolarize, indicating differential, layer-specific roles during stimulus response. Analysis of correlation supports this conclusion, showing anticorrelated activity between superficial and deep layers (Fig. 3h).

We also decomposed VSD fluorescence into excitatory and inhibitory components. For evoked trials, the excitatory component of the signal (>90\%) underwent large deflections in the poststimulus window, far outweighing inhibitory contributions (<10\%) (Fig. 3i). Indeed, the inhibitory fraction remained small throughout both pre- and poststimulus periods. One might expect the inhibitory VSDI fraction to increase in proportion to the excitatory fraction, as it is known that excitatory activity in healthy neocortex quickly recruits a mitigating inhibitory response, preventing runaway excitation (Fino and Yuste, 2011; Isaacson and Scanziani, 2011; Kapfer et al., 2007; Silberberg and Markram, 2007). We therefore analyzed the timecourse of mean membrane potential changes in the inhibitory populations of each layer, revealing that those in superficial layers were significantly hyperpolarized following stimulation, while those in deep layers were significantly depolarized (Fig. 3j,k). Since the dendrites of inhibitory cells tend to be spatially confined (Fig. 1b), those located in deeper layers are unlikely to 


\section{Fig. 3: Fractional contributions to VSDI measurements by layer and cell-type}

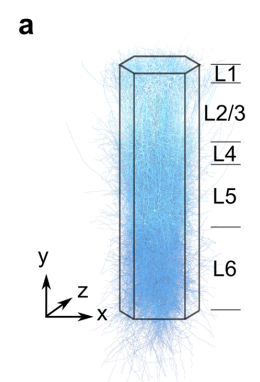

\section{b}

C

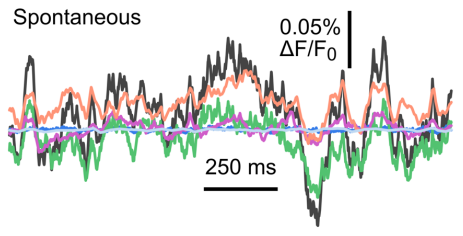

f Evoked
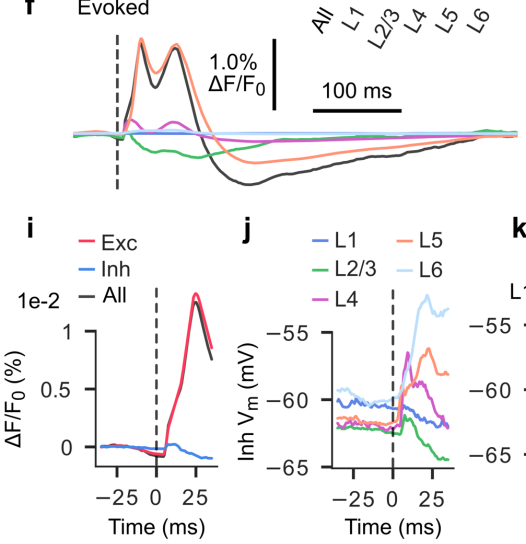

\begin{abstract}
j
\end{abstract}
$\begin{array}{ll}-\mathrm{L} 1 & -\mathrm{L} 5 \\ -\mathrm{L} 2 / 3 & -\mathrm{L} 6\end{array}$

$-\mathrm{L} / 3,-\mathrm{L} 6$
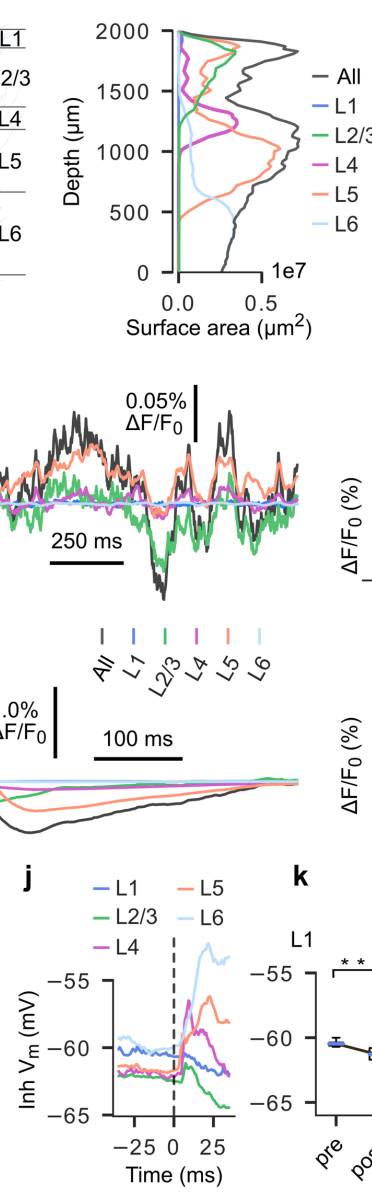

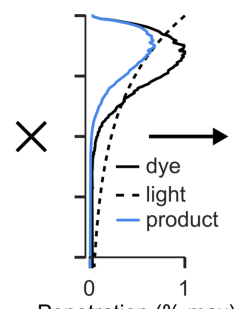

Penetration (\% max) Effective area $\left(\mu \mathrm{m}^{2}\right)$

d

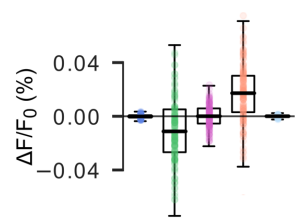

g

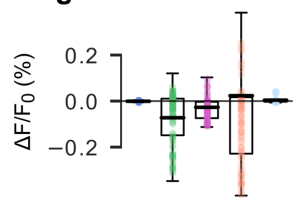

$\mathbf{k}$

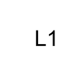

$\mathrm{L} 1 \quad \mathrm{~L} 2 / 3$

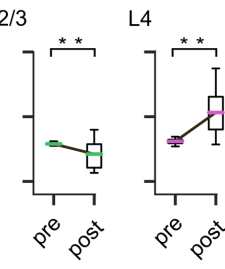

e

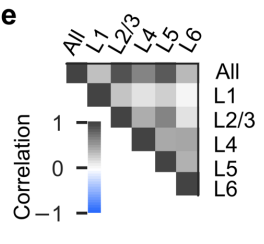

h

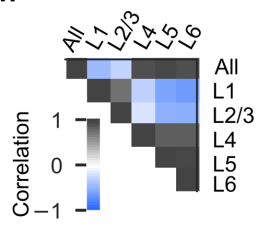

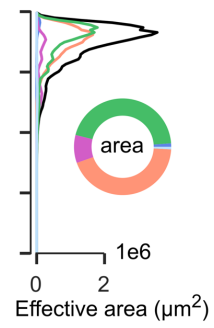

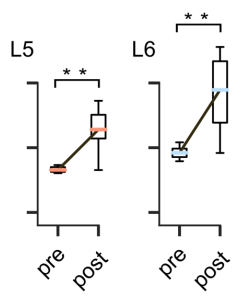

a-b, Surface area contributions for each layer by depth. a, Microcircuit, for reference respecting relative layer positions and axis orientation. $\mathbf{b}$, (Left) Raw (unscaled) neurite surface area profiles by depth for each layer (20 $\mu \mathrm{m}$ bins). (Middle) Depth-dependent scaling prefactors accounting for dye diffusion (solid black line) and light penetration (solid blue line). Solid blue line indicates product. (Right) Effective surface area profiles by depth for each layer (raw surface area $\mathbf{b}$ scaled by product of light attenuation and dye diffusion prefactors (dashed line, middle panel), $20 \mu \mathrm{m}$ bins). c, Spatially-averaged VSD signal (black) with fractional contribution of each layer (colored) for 1.5 seconds of spontaneous activity. d, Boxplot of fractional layer-wise contribution data in c, illustrating overall spread and polarity of each layer's contributions. e, Correlation matrix for all traces in d. f, Spatially-averaged VSD signal (black) with fractional contribution of each layer (colored) for $500 \mathrm{~ms}$ of evoked activity. Plot begins at $-50 \mathrm{~ms}$, stimulus delivered at $0 \mathrm{~ms}$ (dashed line). g, Same as in c, but for evoked activity. $\mathbf{h}$, Same as in $\mathbf{d}$, but for evoked activity. i, Fractional contributions of excitatory and inhibitory populations to overall VSD signal, shown over a $50 \mathrm{~ms}$ second window spanning $25 \mathrm{~ms}$ pre- and $25 \mathrm{~ms}$ poststimulus. j, Mean membrane potentials computed for inhibitory cell populations in each layer, plotted over the same time window as in i. $\mathbf{k}$, Boxplots depicting the difference between pre- and poststimulus membrane potentials for inhibitory cell populations in each layer. Boxplots in each panel were calculated using the $25 \mathrm{~ms}$ pre- and poststimulus periods referred to in $\mathbf{i}$ and $\mathbf{j}$. 
contribute appreciably to the VSDI signal as their morphologies do not extend to a height reachable by dye and light. Therefore, the VSDI signal only "sees" the contributions of hyperpolarized superficial inhibitory neurons.

\section{Disentangling the impacts of sub- and suprathreshold neural activity on VSDI}

It is thought that APs are too brief and too asynchronous to contribute substantially to the VSDI signal, despite causing large fluctuations in $V_{m}$ (Berger et al., 2007; Civillico and Contreras, 2012; Ferezou et al., 2006; Grinvald and Hildesheim, 2004; Petersen et al., 2003a, 2003b). This conclusion is based on simultaneous VSDI and single cell patch-clamp recordings, of which spike-triggered averaging exposes the absence of individual AP waveforms from the VSDI signal (Ferezou et al., 2006). However, such experiments leave open the possibility that large volleys of spikes occurring within a narrow time window could still contribute to the signal. To isolate the effects of spikes on the optical response, we ran our VSDI pipeline on spike-filtered neurite compartment voltage data and compared with unfiltered data (Fig. 4a). Assuming the null hypothesis that VSDI primarily reflects subthreshold activity, we considered any difference between the raw and spike-filtered signals as "noise" due to spikes. This allowed us to calculate a signal-to-spike ratio (SSR), defined in analogy to the signal-to-noise ratio, as the squared quotient of the root mean square (RMS) amplitudes of the unfiltered signal and spiking component. That is,

$$
\begin{gathered}
A=\sqrt{\frac{1}{\Delta t} \int_{t_{0}}^{t_{1}}(\operatorname{VSD}(t))^{2} d t} \\
\mathrm{SSR} \triangleq\left(\frac{A_{\text {raw }}}{A_{\text {raw-filt }}}\right)^{2}
\end{gathered}
$$

which we represented as a continuous variable by binning into $40 \mathrm{~ms}$ intervals with overlapping windows. Conservatively, we estimate that typical VSDI experiments have an SNR of $\sim 10$ (Civillico and Contreras, 2005; Grinvald et al., 1999; Jin et al., 2002; Lippert et al., 2007; Tsau et al., 1996; Zhou et al., 2007). Therefore, when SSR is less than 10 (i.e., less than the empirical SNR of typical experiments), the component of the VSDI signal due to spikes is larger than contamination due to other noise sources, and in principle could be detected. Although SSR did dip slightly below our estimated detectability threshold during the poststimulus window, this is unlikely to be meaningful in most laboratory settings. However, in cases where exceptionally high SNR is achieved, information regarding the spiking component of the VSDI signal may become relevant. Therefore, we sought to understand how the frequency content of spike noise is affected by stimulation (Fig. 4b). A power spectral density analysis of spike noise immediately pre- and poststimulus showed that the frequency 
content differs significantly only below $\sim 100 \mathrm{~Hz}$, with lower frequencies exhibiting greater divergence. Measurements sensitive enough to detect a spiking contribution to the VSDI signal, therefore, would only contain spike-related information below this frequency cutoff and would be dominated by low frequency components. It is important to acknowledge that (as reported previously) contributions of individual spikes are not detectable in mesoscale recordings; rather, it is the aggregate influence of population spiking that adds a small DC offset (and low frequency oscillations) to the VSDI signal, as described above. Assuming a high SNR scenario, we also undertook an analysis of the relative contributions of forward- and backward-propagating APs to the spike-related VSDI signal component (Supplementary Fig. 3). We found that nearly all of the spike-related VSDI signal is due to backwardpropagating APs in dendritic arbors.

\title{
Fig. 4: Detectability of spiking activity in the VSDI signal
}
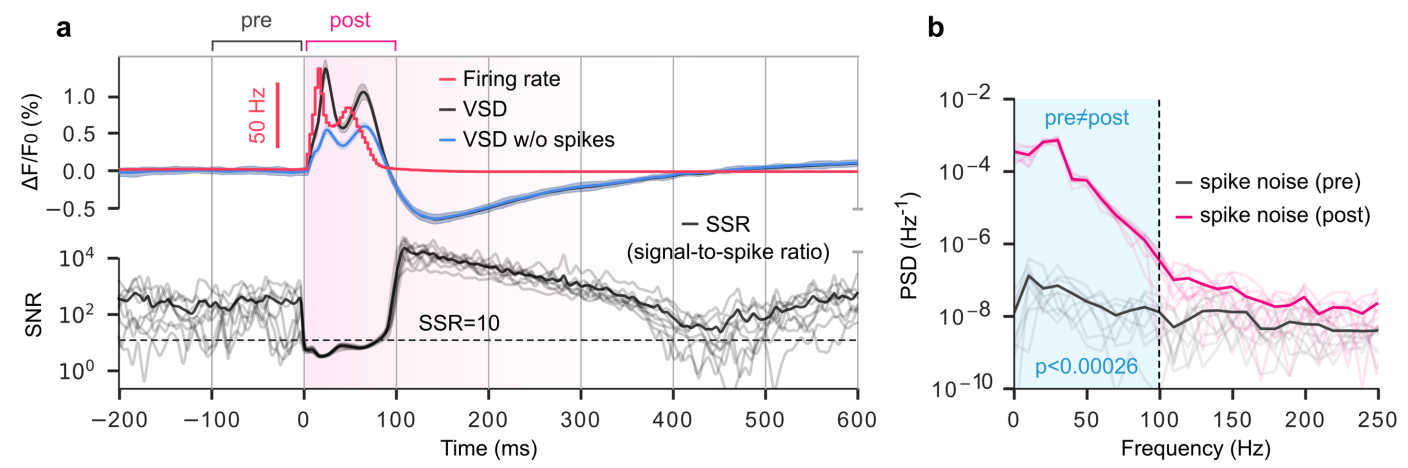

\begin{abstract}
a, Top: signal-to-noise ratio. Noise here refers to "spike noise", or contamination of putative subthreshold measurements by APs. Dashed black line: signal-to-noise ratio $=10$. Bottom: Firing rate (red), VSD (black), and VSD computed excluding spikes (membrane potentials thresholded at $-55 \mathrm{mV}$, blue) for $200 \mathrm{~ms}$ of spontaneous activity followed by $600 \mathrm{~ms}$ of evoked activity. b. Power spectral density of spike noise computed for $100 \mathrm{~ms}$ prestimulus window (black), and $100 \mathrm{~ms}$ poststimulus window (magenta). Blue shaded box indicates frequencies for which pre- and poststimulus noise are significantly different. Dashed black line indicates frequency at which prestimulus noise and poststimulus noise are no longer meaningfully different (paired t-test, significance threshold $=0.01$, adjusted to $2.6 e-4$ for multiple comparisons using Holm-Bonferroni correction).
\end{abstract}

\section{VSDI signals anticorrelate with population firing rates}

In light of the unanticipated observation that spiking activity precedes VSD deflections during evoked activity (Fig. 2c,d), and the interpretation of VSDI as a measure of subthreshold $V_{m}$, we were motivated to inspect the relationship between population firing rates and mean $V_{m}$ in simulations of spontaneous activity. Surprisingly, this revealed a strong inverse association (Pearson correlation coefficient of - 
0.86, $\mathrm{R}^{2}$ value of -0.83 ) between the two at a temporal lag of $\sim 23 \mathrm{~ms}$, with spikes preceding $V_{m}$ (Fig. $5 a, b)$. The relationship between the VSDI signal and spiking exhibited a similar, albeit weaker trend, as expected in view of the correspondence between mean $V_{m}$ and VSD fluorescence (Fig. 5c). Thus, we sought an interpretable explanation for this phenomenon in terms of population dynamics. To this end, mean-field theory was a natural choice as it provides an analytical framework for understanding the dynamics of neural populations, where each population is represented in aggregate as a single unit (Muller et al., 2007; Rudolph et al., 2004; Zerlaut et al., 2018). Since VSDI reports a summary of $V_{m}$ across many neurons, we began by considering the relationship between mean $V_{m}$ and conductance. Mean-field calculations for a conductance-based leaky integrate-and-fire neuron yield an "effective" membrane potential $\left(V_{\text {eff }}\right)$, which approximates the population mean as a function of synaptic bombardment (Rudolph et al., 2004, 2005; Zerlaut et al., 2018) (see Methods). Intuitively, $V_{\text {eff }}$ may be thought of as a noisy short-term prediction of $V_{m}$. In a hypothetical a scenario where conductances are static, $V_{\text {eff }}$ is the value $V_{m}$ converges to by exponential relaxation dynamics.

We proceeded in the calculation of $V_{\text {eff }}$ by isolating firing rates in excitatory and inhibitory subpopulations. Experimental evidence supports the notion that excitatory and inhibitory synaptic currents are tightly balanced in healthy cortical tissue both in the resting state and during sensory processing (Denève and Machens, 2016; Sengupta et al., 2013; Zhou and Yu, 2018). Tight balance of synaptic currents implies that: 1) excitatory and inhibitory firing rates fluctuate synchronously, and 2) inhibitory conductances are greater than excitatory (Sengupta et al., 2013). Consistent with this view, we observed that excitatory and inhibitory firing rates (and thus also excitatory and inhibitory conductances) were highly correlated (Fig. 5d). Additionally, inhibitory conductances were larger than excitatory both in terms of mean and variance, with $a\left\langle g_{i}\right\rangle /\left\langle g_{e}\right\rangle$ ratio of $\sim 9$. Despite the preponderance of inhibitory conductance, we determined that $V_{\text {eff }}$ was affected roughly in equal proportion per unit change in either conductance type (Fig. 5e). Therefore, given its much higher variance, change in inhibitory conductance was the primary driver of $V_{m}$ fluctuations. Finally, since network balance requires high correlation between $\left\langle g_{e}\right\rangle$ and $\left\langle g_{i}\right\rangle$, it follows that mean $V_{m}$, and by extension the VSDI signal, anticorrelate with total firing rate. Next, to confirm our supposition that network balance is a requirement for the aforementioned effect, we performed a series of simulations of spontaneous activity in which network connections (and thus recurrent connectivity) were disabled. Instead, each neuron was fed excitatory and inhibitory input spike trains recorded from previous simulations of the same microcircuit. Importantly, the excitatory and inhibitory inputs were derived from simulations with different random seeds, abolishing the coupling between excitation and inhibition (Fig. $5 \mathrm{~g}$ ), but 


\section{Fig. 5: Population firing rate anticorrelates with VSDI fluctuations}

a

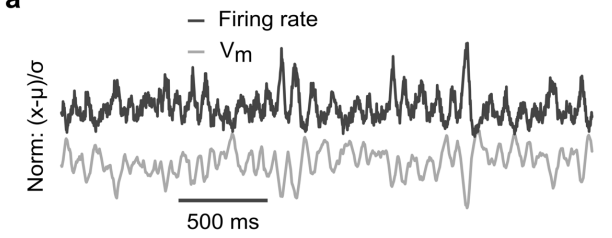

d

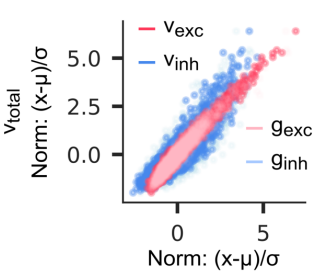

g

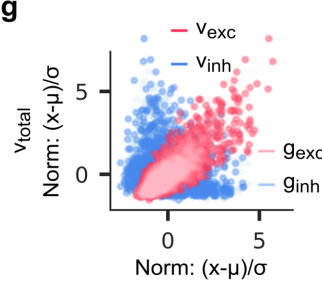

e

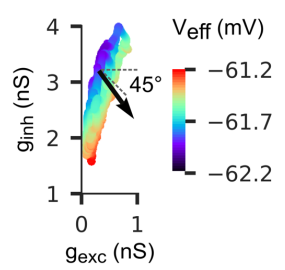

h

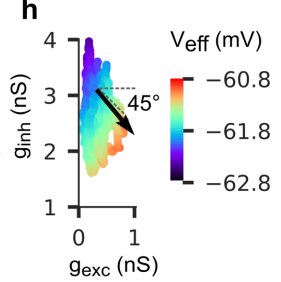

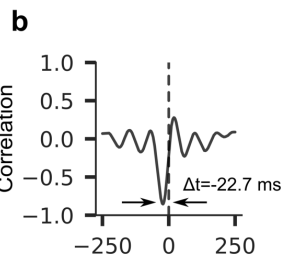

f
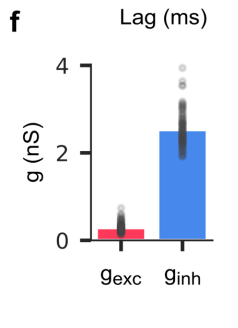

i

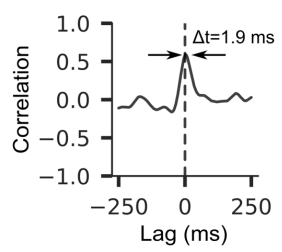

C Firing rate

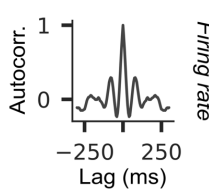

VSD
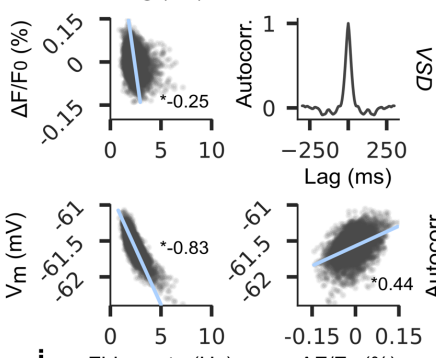

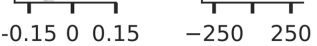

j Firing rate $(\mathrm{Hz}) \quad \Delta \mathrm{F} / \mathrm{FO}(\%) \quad \operatorname{Lag}(\mathrm{ms})$

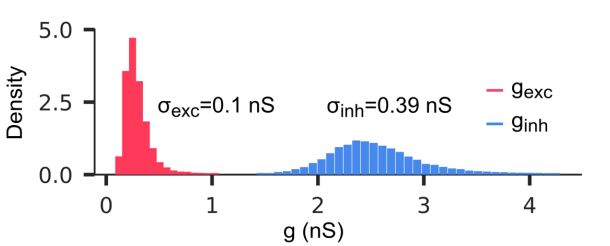

a, Population firing rate (top, dark gray) and mean membrane potential (bottom, light gray); normalized units. Time-shifted by $22.7 \mathrm{~ms}$ to align signals at peak anticorrelation. b Cross-correlogram of population firing rate and mean membrane potential. c, Lower triangular scatter plot matrix depicting correlations between firing rate, VSD, and membrane potential. Starred values indicate R-squared values for linear fit. Diagonal shows autocorrelation. d, Scatter plot of inhibitory and excitatory conductances and firing rates (light blue, pink, blue, red, respectively) against population firing rate; normalized units. e, Scatter plot of excitatory vs. inhibitory conductances. Color reflects corresponding mean field-predicted membrane potential at each point in plot. Black arrow indicates directional gradient (increasing membrane potential). f, Bar plot of mean excitatory and inhibitory conductance values, with individual data points overlaid in black. $\mathbf{g}$, Same as in d, but for decoupled network (i.e., each cell receives only pre-recorded synaptic inputs, and does not influence the network with its output). $\mathbf{h}$, Same as in $\mathbf{e}$, but for decoupled network. i, Cross-correlogram of population firing rate and mean membrane potential for decoupled network. $\mathbf{j}$, Histogram of conductance values in $\mathbf{f}$. Normalized so that AUC $=1$ for both cell-type populations.

preserving the distributions of $\left\langle g_{e}\right\rangle$ and $\left\langle g_{i}\right\rangle$, and therefore also their relationship to $V_{m}$ (Fig. 5h). However, the removal of recurrent network connections inverted the correlational and causal relationship between spiking output and $V_{m}$, as indicated by a flip in the sign of the peak correlation magnitude and its associated lag time, respectively (Fig. 5i). In the absence of network recurrence, presynaptic inputs potentiate spike firing by pushing neurons closer to AP threshold, without subsequent feedback inhibition. We conclude that VSDI primarily reflects subthreshold $V_{m}$ changes associated with inhibitory feedback during spontaneous activity in balanced, recurrently connected cortical networks. 


\section{Extrinsic synaptic inputs decrease the synaptic conductance ratio}

In biological cortex, cellular assemblies are subject to extensive innervation by intracortical and thalamocortical projections, and by long-range projections emanating from white matter tracts (DeFelipe et al., 2002; Gil et al., 1999; Kawaguchi, 2017; Tomioka et al., 2005). Estimates of synapse counts in rat hindlimb somatosensory cortex have been reported as high as 18,000 per neuron (DeFelipe et al., 2002). Because placement of synapses in our microcircuit is constrained by the anatomical apposition of dendrites and axons (Markram et al., 2015; Reimann et al., 2015), only the formation of local synapses ( 1,145 synapses per neuron, on average) is possible, excluding significant innervation arriving from white matter. Thus, each neuron in the NMC receives a tonic injection of depolarizing current $\left(I_{\text {clamp }}\right)$ at its soma to compensate for missing excitatory inputs. This current is the sum of a DC component, which we compute as a percentage of rheobase (the minimal step current required to depolarize the cell to AP threshold), and a Gaussian noise component of small amplitude (Markram et al., 2015). Expressed as a percentage of rheobase, $I_{\text {clamp }}$ is held constant over the entire circuit, though absolute amplitudes vary for each neuron. As a consequence, average conductance values are more than one order of magnitude smaller than those reported in vivo $\left(g_{i}=70.67 \pm 45.23\right.$ $\mathrm{nS}, g_{e}=22.02 \pm 37.41 \mathrm{nS}, g_{i} / g_{e}=14.05 \pm 12.36$ as measured in ketamine-xylazine anesthetized feline cortex during brainstem stimulation (Rudolph et al., 2005); cf. Fig. 5f), with concomitant reductions in variance.

We were curious to understand how the injection of current could affect the results of the previous section, and network dynamics more broadly. To this end, we attempted to replicate naturalistic conditions through the simulation of additional synaptic inputs. Assuming that most inhibitory connections occur locally (<500 $\mu \mathrm{m}$ ) (Fino and Yuste, 2011; Karnani et al., 2014; McDonald and Burkhalter, 1993), we duplicated existing excitatory synapses (5x per synapse, Fig. 6a) on a small handful of L5 PCs $(n=10)$, and set $I_{\text {clamp }}$ to zero. Since it is known that neurons form multisynapse connections (Deuchars et al., 1994; Frick et al., 2008; Markram et al., 1997; Silberberg and Markram, 2007; Silver et al., 2003; Wang et al., 2002), duplicated synapses for each neuron were randomly partitioned into functional groups that received identical input, representing a single connection. Group size was drawn from the distribution of synapses per connection for that cell type. Synaptic inputs consisted of spike trains generated by a homogeneous Poisson process with rate $\lambda$, which we varied incrementally until the root-mean-square error between the number of spikes in a 4 second interval in the new (added synapses) and old (somatic depolarization) simulations was minimized (Fig. 6b,c). We observed that for additional Poisson inputs at the optimal rate $(0.45 \mathrm{~Hz})$, mean excitatory conductance 
Fig. 6: Effects of long-range excitatory synaptic inputs in the absence of somatic depolarization

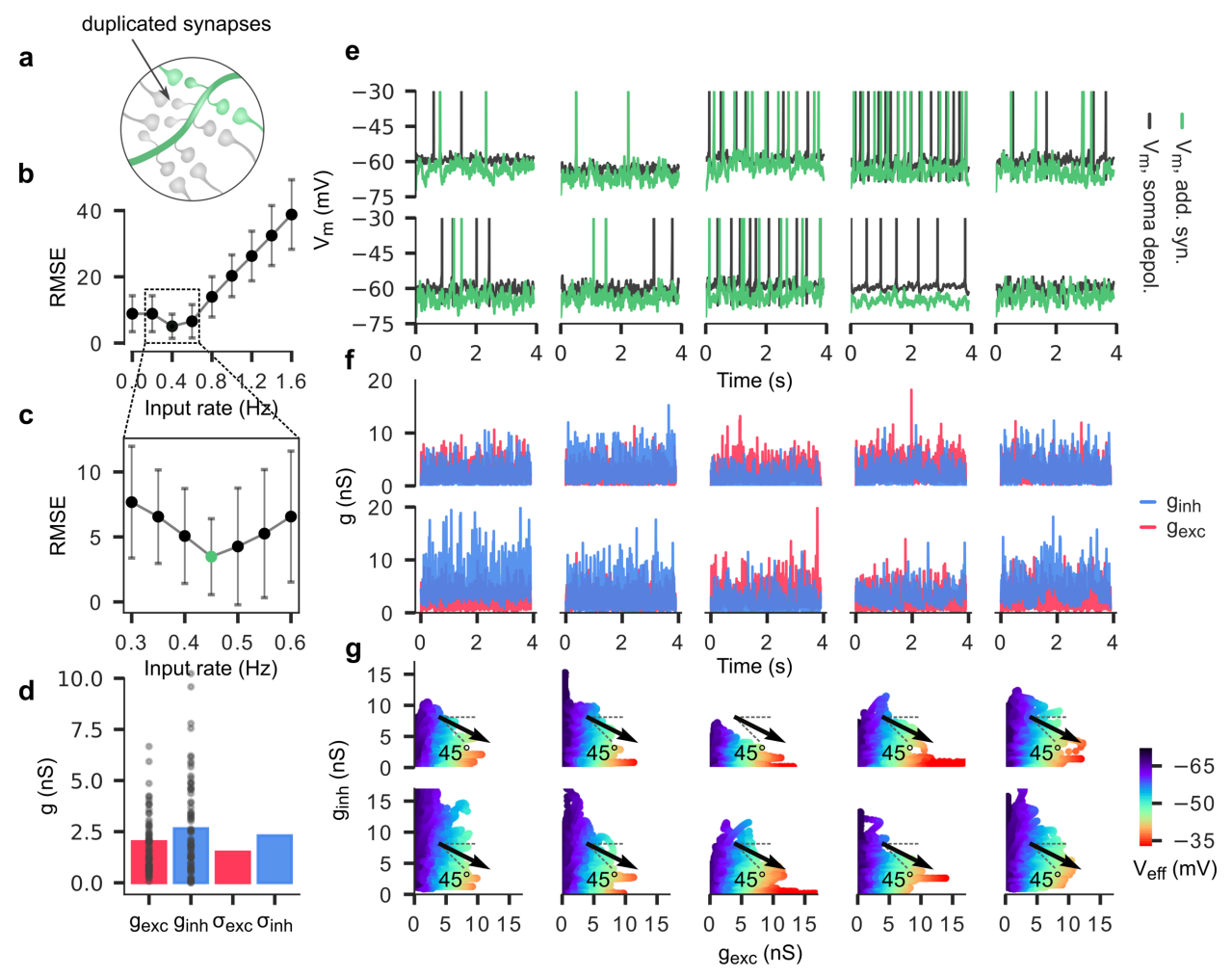

a, Schematic showing duplication of excitatory synapses. Four identical, spatially co-located copies of each excitatory synapse were added to 10 randomly selected L5 PCs in the microcircuit. $\mathbf{b}$, Simulated Poisson inputs were generated at different Poisson rates to evoke postsynaptic spiking. Poisson rates of the added synapses were varied to minimize the RMSE in the difference between the spiking rate of the target postsynaptic cell with and without additional synapses (but with somatic depolarization). $\mathbf{c}$, Expanded view of $\mathbf{b}$, for input Poisson rates between 0.3 and $0.6 \mathrm{~Hz}$. Minimal RMSE indicated in green $(0.45 \mathrm{~Hz})$. d, Mean excitatory and inhibitory synaptic conductances and conductance standard deviations for all 10 neurons (pooled) for 4 seconds of spontaneous activity. e, Voltage trace overlays for each of the 10 neurons for 4 seconds of spontaneous activity. Green: added synapses $(0.45 \mathrm{~Hz})$, no somatic depolarization. Black: somatic depolarization, no additional synapses. f, Excitatory and inhibitory conductances (red and blue, respectively) for each of the 10 neurons for 4 seconds of spontaneous activity with added synapses at $0.45 \mathrm{~Hz}$. g, Scatter plot of excitatory vs. inhibitory synaptic conductances. Color reflects corresponding mean field-predicted effective membrane potential at each point in plot. Black arrow indicates directional gradient (increasing membrane potential).

increased by a factor of $\sim 7$, while inhibitory conductances remained largely unchanged ( $\sim 5$ increase). However, conductance standard deviation changed considerably for both excitatory and inhibitory channels, increasing by a factor of 14.3 and 5.8 , respectively (Fig. $6 \mathrm{~d}$ ). We also examined the relative influence of excitatory and inhibitory conductances in determining $V_{m}$ fluctuations and found that the increased variance in $g_{e}$ corresponded to relatively more influence than $g_{i}$ on the trajectory of $V_{m}$ (Fig. $6 \mathrm{~g}$ ). However, the addition of synapses did not alter the previous observation that a unit change in 
either $g_{e}$ or $g_{i}$ affected $V_{m}$ equally. In summary, a five-fold increase in excitatory synapses with Poisson inputs in the absence of somatic depolarization tended to increase the mean of $g_{e}$ and its influence on $V_{m}$, and dramatically increased the variance of both $g_{e}$ and $g_{i}$.

\section{Discussion}

We constructed a bottom-up, biophysically detailed model of VSDI in a digital reconstruction of rodent somatosensory cortex to relate cellular anatomy and physiology to mesoscale signals, and to seek novel insights regarding cortical dynamics. As a first step, we considered VSDI measurements of evoked responses in our model and found that they were qualitatively and quantitatively similar to analogous experiments in vivo. Next, we used our model to deconstruct the VSDI signal into layer and cell type contributions, revealing context-dependent, strongly differentiated roles for layers 2/3 and 5 . We also examined the influence of spiking activity, and found that while individual spikes are not reflected in VSDI data, large volleys of semi-synchronous spikes could affect measurements.

Furthermore, our model led us to the surprising observation that the VSDI signal anticorrelates with population firing rate with a peak at a lag of $\sim 23 \mathrm{~ms}$. Using a mean-field approach, we discovered that this is due to a predominance of inhibitory conductances, which are coupled to population spiking via recurrent connections. Finally, we considered the effects of including additional excitatory synapses to compensate for missing non-local inputs and found that a Poisson model of spiking inputs led to increased variance in values of conductance. Several details pertaining to the above results merit further discussion.

\section{Lateral spread dynamics of VSDI signals}

As stated previously (see Results), the VSD fluorescence response to TC stimulation underwent nonuniform lateral expansion, wherein the signal quickly saturated near the location of stimulus delivery before gradually extending across the entire microcircuit (Fig. 2f,g). This observation is consistent with in vivo optical responses to evoked activity, in which VSDI signals saturate in a locally confined region near the stimulation site within the first 10-20 ms before expanding further (Civillico and Contreras, 2006, 2012; Fehérvári et al., 2015; Petersen, 2007; Petersen et al., 2003b). Fehérvári et al. (2015) report similar fluorescence dynamics in an in vivo VSDI study of mouse primary visual cortex (V1). In particular, they find that in a localized region around the site of an applied $50 \mu \mathrm{A}$ current impulse, fluorescence rapidly increases within $\sim 10 \mathrm{~ms}$, before saturating and then expanding laterally. They propose that the initial peak primarily reflects monosynaptic excitatory postsynaptic potentials (EPSPs), 
which are followed by the propagation of disynaptic activity at greater latencies. This explanation is consistent with our finding that the first response occurs locally and quickly plateaus, since it is likely due to feedforward PSPs evoked by direct TC innervation at the center of the microcircuit. Subsequent activity spread would occur only following a monosynaptic delay, as the targets of TC projections propagate the signal to their postsynaptic partners. Also, we note that in comparison to average signal transmission speeds reported in literature (Table 1), the wavefront phase velocities calculated here are relatively low. We speculate that this may be due to slicing of mid-range intracortical axons during the morphology reconstruction process. It is well known that extended axonal arbors are at risk of slicing during histological processing, and efforts were made to repair severed arbors using statistical methods (Markram et al., 2015). However, it is unlikely that such repairs would fully correct for slicing artifacts, leaving open the possibility that significant numbers of mid-range connections are missing. If true, it would tend to decrease wavefront propagation speeds, as signal transmission would be forced to proceed strictly through short-range connections.

\section{Lack of correlated activity between VSDI signals and layer 2/3 PCs}

A point of disagreement between our results and those described in literature is the degree to which VSDI recordings are correlated with simultaneous whole-cell (WC) recordings in L2/3 (see Supplementary Fig. 1). Several in vivo studies have reported a high correlation between VSD fluorescence and the $V_{m}$ of single neurons in L2/3 rodent barrel cortex (Berger et al., 2007; Ferezou et al., 2006; Petersen et al., 2003a, 2003b). However, due to the technical challenges associated with simultaneously performing VSDI and WC recordings in live animals, these studies used anesthesia (Ferezou et al., 2006; Petersen et al., 2003a, 2003b) or in vitro slice preparations (Berger et al., 2007) to establish the correspondence between $V_{m}$ and VSDI traces. It has been shown that anesthetic agents increase cortical synchrony and pairwise neural correlations (Antkowiak, 2002; Greenberg et al., 2008; Kreuzer et al., 2010; Murphy et al., 2011). Of particular relevance, Greenberg et al. (2008) found that correlated AP firing in pairs and populations of $L 2 / 3$ neurons in rat visual cortex increased significantly during anesthesia as compared to the awake state. Therefore, the disparity between the strength of VSDI- $V_{m}$ correlations observed in vivo and those extracted from our simulations may be at least partly explained by differences in cortical state. Since VSDI signals reflect an average over $V_{m}$ deflections in a large number of neuronal processes mostly situated in L2/3, anesthesia-induced synchrony among L2/3 neurons would tend to increase the correlation between any given L2/3 neuron and the population mean. Our model does not consider the effects of anesthesia, nor do we observe the emergence of oscillatory cortical states. Thus, both pairwise and population neural correlations 
remain relatively weak during spontaneous activity, resulting in a lower correspondence between VSD fluorescence and individual $V_{m}$ measurements.

\section{Changes in spiking activity precede deflections in mean membrane potential}

We showed that spikes precede $V_{m}$ fluctuations during both spontaneous and evoked activity (see Fig. 2c,d; Fig. 4a; Fig. 5b), confirming several studies including one in rat barrel cortex (Petersen et al., 2003b), and two others in ferret visual cortex (Eriksson et al., 2008; Roland et al., 2006). A reasonable expectation may be that, on the contrary, increases in VSD fluorescence should precede increased spike firing, since membrane depolarization would tend to bring neurons closer to threshold making APs more likely. However, as suggested by Eriksson et al. (2008), since each cell contacts many postsynaptic partners (452 \pm 272 in our microcircuit), any given AP will elicit postsynaptic potentials (PSPs) in hundreds to thousands of other cells, meaning that a mere handful of spikes can significantly impact mean $V_{m}$ in a population. Of course, spike initiation requires membrane depolarization, but only a fraction of the population is active at once ( $26 \%$ at evoked response peak, and $\sim 0.4 \%$ during baseline; $2 \mathrm{~ms}$ bins). Therefore, $V_{m}$ changes associated with spike firing are outweighed by downstream PSPs, with a monosynaptic delay. We found a $6.9 \mathrm{~ms}$ delay between peak spiking and subthreshold response to stimulation (Fig. 2c,d), and a $22.7 \mathrm{~ms}$ delay during spontaneous activity (Fig. 5b). Monosynaptic signal transmission reportedly requires between 6 and $14 \mathrm{~ms}$ in cortex (GonzálezBurgos et al., 2000), suggesting that deflections in mean $V_{m}$ primarily reflect monosynaptic activity in the first case (evoked), and disynaptic inhibition in the second (spontaneous). Indeed, this conclusion is supported by the reversal of sign in the correlation between $V_{m}$ and firing rate in the putatively disynaptic, spontaneous case. Furthermore, uncoupling the network, thereby disabling recurrent disynaptic connections, abolished the temporal lag and inverse relationship between spiking and mean $V_{m}$ (Fig. 5i), creating a situation in which increased membrane potential merely potentiates APs. Thus, VSDI may report either monosynaptic excitation or disynaptic inhibition depending on the presence or absence of external inputs. That this aspect of VSDI measurements could have been missed in previous research begs explanation. First, simultaneously performing VSDI measurements and population spike recordings is a technical challenge, and has only been attempted in a handful of studies (e.g., (Eriksson et al., 2008; Roland et al., 2006)). Moreover, the lag between VSDI signals and spike firing, in addition to any extracortical noise sources, would tend to obscure the immediate observation of a correlation. Last, VSDI signals are imperfect proxies of mean $V_{m}$ since they are heavily biased towards contributions of neurites within L2/3 (Fig. 2b). 


\section{Influence of cortical state on network dynamics}

Regenerative activity in the microcircuit is sensitive to the concentration of extracellular calcium $\left(\left[\mathrm{Ca}^{2+}\right]_{0}\right)$ and level of tonic depolarization. As demonstrated by Markram et al. (2015), increasing tonic depolarization has the effect of pushing cells closer to AP threshold, while decreasing $\left[\mathrm{Ca}^{2+}\right]_{0}$ (within the physiological range, 1-2 $\mathrm{mM}$ ) tends to shift the excitatory-inhibitory balance in favor of inhibition. Varying these two parameters, they observed the emergence of four distinct regimes in the behavior of the microcircuit, characterized by the presence or absence of regenerative activity for either spontaneous or evoked conditions. Furthermore, within each regime, varying $\left[\mathrm{Ca}^{2+}\right]_{0}$ moved the network along a spectrum between synchrony (high $\left.\left[\mathrm{Ca}^{2+}\right]_{0}\right)$ and asynchrony (low $\left.\left[\mathrm{Ca}^{2+}\right]_{0}\right)$. It is well established that in vivo concentrations of extracellular ions are maintained within relatively narrow physiological ranges by tightly regulated homeostatic pathways, and that alterations in these concentrations affect network dynamics (Barreto and Cressman, 2011; Ding et al., 2016; Gleichmann and Mattson, 2010; Henn et al., 1972; Kraio and Nicholson, 1978; Rasmussen et al., 2017). We theorize that a spectrum of network regimes similar to those observed in our network could also be present in biological cortex, and that under normal physiological conditions, the network sits at or near the transition point between regimes. This could serve to maximize sensitivity subject to the constraint of avoiding runaway excitation, thereby optimizing the potential of cortical tissue to encode sensory stimuli. Furthermore, it is known from in vivo recordings that cortical neurons in awake animals exhibit low input resistances, relatively depolarized membrane potentials ( -60 mV), and significant $V_{m}$ fluctuations (Destexhe, 2007, 2010; Destexhe et al., 2003). Collectively, these properties are referred to as the "high-conductance state", since they are a consequence of synaptic bombardment causing mean conductances to exceed resting conductance (Destexhe, 2007). High-conductance states are thought to play an important role in determining neural response properties, with consequences for computation (Destexhe, 2007, 2010; Destexhe et al., 2003). Independent and convergent lines of evidence suggest that the critical transition point in our model (somatic depolarization at $\sim 100 \%$ and $\left.\left[\mathrm{Ca}^{2+}\right]_{0}=1.25 \mathrm{mM}\right)$ is most analogous to quiet wakefulness, with some high-conductance state properties.

Comparing the time to peak and half width duration of our evoked VSD fluorescence response to those obtained in a similar study of mouse barrel cortex (Fig. 2b) reveals a strong correspondence in the temporal profile of cortical activation for awake animals (half-width: $86 \pm 69 \mathrm{~ms}$ ), but not anesthetized (37 $\pm 8 \mathrm{~ms}$ ) (Ferezou et al., 2006). Furthermore, the dominance of inhibitory conductances, relatively depolarized membrane potentials $(\sim-60 \mathrm{mV})$, and significant $V_{m}$ fluctuations argue for a high- 
conductance-like state, which is associated with wakefulness (Destexhe, 2007, 2010; Destexhe et al., 2003). Finally, the slow-wave oscillations typically observed during sleep and anesthesia are not present in our model, though this could be a result of missing thalamocortical interactions or neuromodulation, rather than a local property of the tissue (Contreras and Steriade, 1996; Contreras et al., 1997; Mena-Segovia et al., 2008; Murphy et al., 2011; Steriade, 2000). Thus, we propose that the results of our model are best interpreted as representing an awake, in vivo-like state. We note at least one major caveat to the above interpretation, namely that values of synaptic conductances are significantly lower than those estimated from in vivo recordings in the awake state. Since magnitude of conductance in dendritic arbors is known to affect the integrative properties of neurons (Destexhe, 2007; Destexhe et al., 2003), this discrepancy is likely to affect network dynamics. However, we leave a detailed analysis of the consequences for future research.

\section{VSDI-firing rate anticorrelation lag time: an index of locality?}

We have shown that during spontaneous activity, population firing rate anticorrelates with mean $V_{m}$ and spatially averaged VSDI data for recurrently connected balanced networks. However, this observation was made in the context of an isolated local network, suggesting that a relaxation of these conditions could moderate the effect. To this end, we attempted to replicate the effects of extrinsic (non-local) synaptic inputs and assessed changes in the mean, standard deviation, and ratio of $g_{e}$ and $g_{i}$. Due to resource constraints, we were restricted to NMC simulations with additional extrinsic inputs at a mere handful of synapses in the network, and were thus unable to evaluate alterations to the relationship between mean $V_{m}$ and mean firing rate as a function of long-range input statistics. However, the preliminary results indicate a shift in the mean conductance ratio in individual neurons, suggesting that extrinsic inputs can influence local network properties. Indeed, Roland (2017) suggests that network balance is a local property that is tightly maintained during spontaneous activity, but which may be temporarily disrupted by a quick succession of excitatory APs from non-local cortical regions. For sufficiently powerful inputs, local inhibitory neurons cannot fully compensate for increased excitatory activity, and additional inhibitory cells must be recruited to prevent runaway excitation (Dehghani et al., 2016; Huys et al., 2016; Roland, 2017). The process of recruiting additional inhibition may introduce a delay between peaks in inhibitory and excitatory firing, relaxing the tight balance of activity in the network. Thus, we propose that the inverse relationship between VSDI signals and mean firing rate revealed by our model could provide an "index of locality", i.e. an increased delay in the peak anticorrelation between VSDI and population spiking represents a stronger perturbing extrinsic influence. Mechanistically, the delay between changes in firing rate and VSDI fluctuations reflects the 
time required after a spike is fired for recurrently connected inhibitory neurons to respond by hyperpolarizing the $V_{m}$ of the spiking cells. We predict that stronger peripheral inputs or corticocortical interactions will result in greater lag times for peak anticorrelation between VSD fluorescence and population firing rates. Indeed, a preliminary analysis of the evoked response to TC stimulation (see Supplementary Fig. 5) revealed a significant shift in the anticorrelation lag time as compared with spontaneous activity (23 ms vs. $130 \mathrm{~ms}$ ), consistent with this prediction. Currently, the bulk of evidence for network balance has been drawn from correlations in the membrane potentials of nearby cells with similar orientation tuning, and measurements of conductance ratios over time in individual cells (Denève and Machens, 2016). Our proposal for the novel use of VSDI data to determine a locality index would add confirmatory evidence for the balance of inhibition and excitation at the network level, while simultaneously providing a metric for evaluating the degree of influence of non-local activity on local microcircuitry. We leave a thorough exploration of this hypothesis and its implications for future studies.

\section{Limitations and outlook}

As regards the validity of the in silico model of VSDI presented here, we propose the consideration of three conceptually distinct layers: 1) whether the biophysical model of VSDI, i.e. the calculations linking cellular activity to measured fluorescence, reasonably approximate reality, 2) whether the composition and architecture of the tissue itself is biologically plausible, 3 ) whether the simulations are functionally representative of biological neocortex.

On the first account, we assert that the excellent linearity and fast kinetics of VSDs (Lippert et al., 2007) greatly simplify their analytical relationship to $V_{m}$. Regarding the second concern, a caveat is the absence of several important structural details from the version of the NMC used here, including glial cells, vasculature, and long-range intracortical axons (Markram et al., 2015). However, in the case of glia, the slow timescale of response (3-4 ms (Schummers et al., 2008)) and small amplitude of $V_{m}$ deflections (1-7 mV (Kelly and Essen, 1974)) make it unlikely that they contribute meaningfully to VSDI signals. As regards vasculature, since our in silico VSDI pipeline already accounts for the bulk optical properties of cortical tissue (see Methods), their effects have, in principle, been accounted for. With respect to the third concern above, several details that are likely to influence network dynamics, including gap junctions, multivesicular release, neuromodulation, and synaptic plasticity, are not present in this version of the NMC. 
In addition, we note several caveats concerning the comparison of our in silico stimulation protocol to whisker deflection experiments in rodents. First, as a model of hindlimb somatosensory cortex, the NMC lacks the unique cytoarchitecture and anatomical organization that characterize barrel cortex (Schlaggar and O'Leary, 1994; Simons et al., 1984). Furthermore, the NMC excludes the trigeminal and thalamic nuclei, and therefore does not exhibit sensory processing delays (and VSDI response latencies) or the modulation of cortical dynamics by TC feedback. Previous experiments have shown that cortical activation patterns depend on stimulus strength, with a tendency for excitation evoked by weak stimuli to remain confined to a single barrel (Berger et al., 2007; Fehérvári et al., 2015; Gollnick et al., 2016; Petersen et al., 2003b). In our model, TC projection fibers innervating the geometrical center of the microcircuit fire a simultaneous AP, a construction that does not capture the full complexity of afferent TC signaling nor permit modification of the stimulus strength in a biologically plausible way. Finally, experiments have implicated reciprocal TC pathways (Bazhenov et al., 2002; Hughes et al., 2002; Steriade et al., 1993) and intracortical interactions (Timofeev et al., 2000) in the emergence of slow wave activity. It is known that cortical oscillations interact with sensory responses to produce differentiated VSDI signals (Petersen et al., 2003a). Thus, an in silico account of the effects of brain state on VSDI measurements awaits future iterations of the NMC that include TC feedback and corticocortical interactions.

In view of these concerns, future versions of BBP brain models that extend well beyond the microcircuit scale are forthcoming. An anatomically complete reconstruction of the somatosensory cortex (and ultimately the entire neocortex) featuring biologically appropriate macro- and micro-connectivity (Reimann et al., 2019) will help to resolve questions concerning the effects of missing long-range inputs. Additionally, a model of neuromodulatory dynamics, the effects of which are known to be implicated in the transitions between, and maintenance of, cortical states (Colangelo et al., 2019), will support the investigation of state-dependent VSDI responses. Finally, a detailed model of the neuroglia-vasculature ensemble will pave the way for future simulation-based studies of imaging techniques such as $\mathrm{fMRI}$ that rely on blood-oxygen-level-dependent (BOLD) signals.

\section{Concluding remarks}

This study demonstrates the utility of bottom-up biophysical modeling as a complement to experimental approaches for understanding the relationships between spatial and temporal scales of cortical signaling. Using our model, we clarified which aspects of neural anatomy and physiology shape VSDI signals. Additionally, we discovered that during ongoing spontaneous activity, VSDI primarily reflects 
subthreshold activity associated with recurrent inhibitory connections. These insights were gleaned from in silico data beyond the reach of current experimental techniques.

\section{Methods}

\section{Microcircuit}

Our in silico VSDI model was implemented in a digital microcircuit consisting of a connected network of 31,346 neurons, $\sim 8$ million connections, and $\sim 37$ million synapses. The network was arranged in a columnar volume 462 x $400 \mu \mathrm{m}$ wide, and $2082 \mu \mathrm{m}$ deep. A spatially extended version was constructed by interconnecting 7 such units in a hexagonal tiling (the "mosaic"). In this configuration, depth axes were mutually parallel, and columnar surfaces were coplanar (Fig. 1h). The cell morphologies populating the circuit were obtained from 3D reconstructions of biocytin-stained neurons from juvenile rat hindlimb somatosensory cortex, while the placement, connectivity, and electrophysiological properties of each cell was determined algorithmically and constrained by sparse data derived from experiments and literature (Markram et al., 2015). TC innervation was reconstructed using VPM axon bouton density profiles in rat barrel cortex, and synapses were probabilistically assigned to incoming fibers using a Gaussian distribution centered around each fiber (Markram et al., 2015). See Markram et al. (2015) for additional details concerning microcircuit construction and composition.

\section{Supercomputing}

A 2-rack Intel supercomputer using dual socket, $2.3 \mathrm{GHz}, 18$ core Xeon SkyLake $6140 \mathrm{CPUs}$, with a total of 120 nodes, 348 GB of memory, and 46 TB of DRAM was used to run the simulations and carry out analysis.

\section{Simulation}

Simulations were conducted using proprietary software based on the NEURON simulation environment (Hines and Carnevale, 1997). Data were output in the form of binary files containing spike times and compartment $V_{m}$ sampled every $0.1 \mathrm{~ms}$ for each neuron in the network. Extracellular calcium and potassium concentrations, as well as somatic depolarization are free parameters in the model, and were set to $1.25 \mathrm{mM}, 5.0 \mathrm{mM}$, and $\sim 100 \%$ threshold, respectively, to most closely mimic an in vivo-like network state (Markram et al., 2015). 
Trials simulating evoked responses modeled TC stimulation with of a single pulse of activity in 60 contiguous thalamic fibers projecting to the geometric center of the microcircuit. For experiments requiring a larger cortical surface area, the same approach was applied to the spatially extended hexagonal microcircuit tiling (see Microcircuit above). Activity was simulated over 10 trials (i.e. random seeds) for a duration of 5 seconds each, with the first 2 seconds of data in each trial discarded to avoid any boundary condition-dependent artefacts. The stimulus was delivered at $2500 \mathrm{~ms}$, meaning that each trial consisted of an initial period of $500 \mathrm{~ms}$ of spontaneous activity, followed by $2500 \mathrm{~ms}$ of poststimulus activity.

\section{Signal calculation}

We assumed that the VSDI signal emanating from a small patch of cellular membrane was linearly related to the product of the membrane surface area and $V_{m}$ (Berger et al., 2007; Ferezou et al., 2006, 2009; Gollnick et al., 2016; Grinvald and Hildesheim, 2004; Lippert et al., 2007; Petersen et al., 2003a, 2003b). Our neuronal morphologies are composed of small segments ("compartments") of equipotential cable whose surface area and transmembrane voltage were multiplied to obtain the raw VSDI signal. This raw signal was scaled for each compartment as a function of depth to account for the physics of dye diffusion and the scattering and absorption of illumination light (Fig. 1d). The degree of signal attenuation due to uneven staining through the cortical depth was interpolated from data measured in four mouse brains treated with $\mathrm{RH} 1691$ voltage-sensitive dye, flash-frozen and sliced into $20 \mu \mathrm{m}$ thick cryosections (Ferezou et al., 2006). To reduce data storage requirements, we divided the microcircuit into voxels, within which an aggregate signal was computed by summing the contributions of all compartments in that voxel:

$$
v_{i j k}(\mathrm{t})=\sum_{r \in i j k} \alpha_{r} \Gamma\left(y_{r}\right)\left(\Delta V_{m}^{r}(t)+G_{0}\right)
$$

where $v_{i j k}$ denotes the value in the $i j k^{\text {th }}$ voxel, $\alpha_{r}$ is the surface area of the $r^{\text {th }}$ compartment, $\Gamma\left(y_{r}\right)$ is an attenuation prefactor accounting for dye penetration and scattering/absorption of illumination light at depth $y$ for compartment $r, \Delta V_{m}^{r}$ is the change in membrane potential for the $r^{\text {th }}$ compartment, and $G_{0}$ is a constant reflecting the combined reflecting the combined contributions of background noise and autofluorescence (assumed isotropic). The value of $G_{0}$ was fixed by requiring that a $10 \mathrm{mV}$ change in $V_{m}$ correspond to a $\sim 0.5 \%$ change in fluorescence over baseline $\left(\Delta \mathrm{F} / \mathrm{F}_{0}\right)$, as reported by Ferezou et al. (2006), assuming an average resting potential of $-65 \mathrm{mV}$. 
To model the effects of scattering and absorption in the tissue, we used a Monte Carlo simulationbased approach (see Point Spread Function) to compute an effective point spread function (PSF) for increasing depths along an axis perpendicular to the cortical surface. We used the PSF at each depth to determine the standard deviation of a Gaussian kernel, which we convolved with the horizontal data slice at that depth:

$$
\begin{gathered}
H_{j}=\left(\begin{array}{ccc}
v_{0 j 0} & \cdots & v_{0 j l} \\
\vdots & \ddots & \vdots \\
v_{n j 0} & \cdots & v_{n j l}
\end{array}\right) \\
\widehat{H}_{j}(t)=H_{j}(t) * g\left(\sigma_{j}\right) \\
\widehat{V}=\bigcup_{j} \widehat{H}_{j}
\end{gathered}
$$

$H_{j}$ (Equation. (2.2)) is a horizontal data slice at depth $j$, where $i \in\{0, \ldots, n\}$ and $k \in\{0, \ldots, l\}$. In Equation (2.3), $\widehat{H}_{j}$ is the filtered data slice at depth $j, H_{j}$ is the original data slice, and $g$ is a Gaussian kernel, with depth-dependent standard deviation $\sigma_{j}$. The union of all filtered slices yields the filtered data volume $\widehat{V}$ (Equation (2.4)). Post-convolution, each vertical (j-axis) column of voxels was accumulated into a single value, resulting in a two-dimensional matrix of pixels, which we stored as an image (Equation (2.5)). VSDI signals were computed as a fractional change in fluorescence over resting intensity (Ferezou et al., 2007, 2009; Kleinfeld and Delaney, 1996; Orbach et al., 1985; Shoham et al., 1999). This gives raw and normalized signal intensities for each pixel in an image matrix:

$$
\begin{gathered}
F_{i k}(t)=\sum_{j} \hat{v}_{i j k}(t) \\
V S D_{i k}(t)=\frac{\Delta F_{i k}(t)}{F_{i k}^{0}}-1
\end{gathered}
$$

where $F_{i k}^{0}$ is a baseline fluorescence image obtained by averaging the first 100 frames (50 ms of data sampled at $2000 \mathrm{~Hz}$ ). We used Equation (2.6) to calculate voltage-sensitive dye signals in this work.

\section{Point spread function}

We calculated an empirical, depth-dependent point spread function (PSF) to account for blurring in the final image due to both scattering of emitted fluorescence photons in cortical gray matter, and also 
optical distortions caused by out-of-plane signal. Our method for calculating the PSF consisted of two steps: first, we used a Monte Carlo (MC) simulation-based approach to model the scattering and absorption of photons emitted from a point source within the tissue volume, varying the depth of the source in 50 micron increments; second, we used ray transfer matrix analysis to trace the trajectories of these photons through a tandem-lens optical system onto a sensor at the image plane.

MC simulations were carried out using a proprietary library built on an open-source framework for physical rendering using backward MC ray tracing, the Physically-based Rendering Toolkit (PBRT) (Pharr et al., 2016). We extended the PBRT framework to simulate photon interactions with highly turbid media using forward MC simulations based on an algorithm proposed by Abdellah et al. (2017). To determine the PSF, we moved an isotropically radiating point source of $10^{8}$ photons throughout a semi-infinite (lateral extent) volume of tissue, beginning at the bottom of the microcircuit in increasing increments of $50 \mu \mathrm{m}$, allowing each photon to scatter until it was either absorbed, or exited the cortical surface. Coefficients of reduced scattering and absorption at $\sim 665 \mathrm{~nm}$ were taken to be $4 \mathrm{~mm}^{-1}$ and $0.4 \mathrm{~mm}^{-1}$, respectively, interpolated from optical measurements made in rat gray matter for wavelengths of light spanning 450 to $700 \mathrm{~nm}$ (Mesradi et al., 2013). We chose the wavelength to correspond to peak emissions in the $\mathrm{RH}-1691 / 1692$ family of blue voltage-sensitive dyes (Berger et al., 2007; Ferezou et al., 2006; Petersen et al., 2003a; Shoham et al., 1999). Refraction at the tissue-air interface was calculated using the vector formulation of Snell's law. Using ray transfer matrix analysis, photons emanating from the tissue surface were propagated through an optical system modeled after a tandemlens epifluorescence macroscope setup first proposed by Ratzlaff and Grinvald (1991), and subsequently used in several VSDI studies (Ferezou et al., 2006; Petersen et al., 2003a, 2003b). The system consists of two compound lenses (modeled using the thin lens approximation) set to infinite focus and placed face-to-face (Ratzlaff and Grinvald, 1991). Optical parameters (focal length, f-number and working distance) were taken from Petersen et al. (2003b), resulting in a focal plane $\sim 300 \mu \mathrm{m}$ below the pia. The point source produced a sunburst image pattern on the detector array for each depth, to which a two-dimensional Gaussian surface was fit using a non-linear optimizer (Python). From these surfaces, we extracted the average spatial standard deviation ,and fit the resulting array of values to a decaying exponential function to determine a depth-dependent PSF for the entire tissuelens system. The standard deviations extracted from our PSF were used to calculate spatial kernel widths for convolution of the data with a Gaussian filter (see Equation (2.3)). 


\section{Mean-field equations}

Following the approach of Dorn and Ringach (2003), Kuhn et al. (2004), Muller et al. (2007), and Zerlaut et al. (2018), the membrane potential of a LIF neuron evolves according to:

$$
\begin{gathered}
C_{m} \frac{d V_{m}}{d t}=g_{e}\left(E_{e}-V_{m}\right)+g_{i}\left(E_{i}-V_{m}\right)+g_{l}\left(E_{l}-V_{m}\right)-I_{\text {clamp }} \\
\frac{d g_{e}}{d t}=-\frac{1}{\tau_{e}} g_{e}+q_{e} S_{e}(t) \\
\frac{d g_{i}}{d t}=-\frac{1}{\tau_{i}} g_{i}+q_{i} S_{i}(t)
\end{gathered}
$$

where $C_{m}$ is membrane capacitance, $g_{e}, g_{i}$, and $g_{l}$ are excitatory (AMPA and NMDA), inhibitory (GABA) synaptic conductance, and leak conductance, respectively, $E_{e}, E_{i}$, and $E_{l}$ are the respective reversal potentials, $\tau_{e}$ and $\tau_{i}$ are the excitatory and inhibitory time constants, $q_{e}$ and $q_{i}$ are the quantal synaptic conductance increases, and $S_{e}$ and $S_{i}$ are presynaptic spike trains (Dorn and Ringach, 2003; Kuhn et al., 2004; Muller et al., 2007; Zerlaut et al., 2018). The term $I_{\text {clamp }}$ represents a depolarizing current injected at the soma to compensate for missing external inputs to our microcircuit (see Results). Defining a new quantity, the "effective potential", as:

$$
V_{\mathrm{eff}}=\frac{g_{e} E_{e}+g_{i} E_{i}+g_{l} E_{l}-I_{\text {clamp }}}{\sum_{k} g_{k}}
$$

allows us to rewrite Equation (3.1) in terms of the true and effective potentials, and rearrange to obtain:

$$
\tau_{\mathrm{eff}} \frac{d V_{m}}{d t}=V_{\mathrm{eff}}-V_{m}
$$

where

$$
\tau_{\mathrm{eff}}=\frac{C_{m}}{\sum_{k} g_{k}}
$$

We note that Equation (3.5) has the form of an RC low-pass filter, with a cutoff frequency given by $1 / \tau_{\text {eff }}$; therefore we expect $V_{\text {eff }}$ to be a reasonable approximation of $V_{m}$, provided the frequency of characteristic fluctuations in $V_{\text {eff }}$ don't exceed $1 / \tau_{\text {eff. }}$. The above equations hold true both for a single neuron, and in expectation across a population of neurons. Furthermore, if $S_{e}$ and $S_{i}$ are inhomogeneous Poisson processes, then in expectation, the terms $S_{e}$ and $S_{i}$ in Equations (3.2) and (3.3) are replaced with time-varying mean firing rates (Muller et al., 2007). Thus, in expectation,

$$
\left\langle V_{\text {eff }}\right\rangle=\frac{\left\langle g_{e}\right\rangle E_{e}+\left\langle g_{i}\right\rangle E_{i}+\left\langle g_{l}\right\rangle E_{l}-\left\langle I_{\text {clamp }}\right\rangle}{\sum_{k}\left\langle g_{k}\right\rangle}
$$




$$
\begin{aligned}
& \frac{d\left\langle g_{e}\right\rangle}{d t}=-\frac{1}{\tau_{e}}\left\langle g_{e}\right\rangle+q_{e} v_{e} \\
& \frac{d\left\langle g_{i}\right\rangle}{d t}=-\frac{1}{\tau_{i}}\left\langle g_{i}\right\rangle+q_{i} v_{i}
\end{aligned}
$$

Numerical integration of Equations (3.8) and (3.9) allowed us to analytically relate mean firing rates to $V_{m}$.

\section{Post-processing and analysis}

All code for analysis was written in the Python programming language.

\section{Acknowledgements}

We thank M. Nolte, M. Reimann, G. Chindemi, S. Ramaswamy, C. Colangelo, and other members of the Blue Brain Project for useful feedback and discussions, and C. Favreau for visualization support. The authors are also grateful to J. King, M. Gevaert, and W. Van Geit for technical assistance. This study was supported by funding to the Blue Brain Project, a research center of the École polytechnique fédérale de Lausanne, from the Swiss government's ETH Board of the Swiss Federal Institutes of Technology.

\section{Author information}

\section{Affiliations}

Blue Brain Project, École polytechnique fédérale de Lausanne (EPFL), Campus Biotech, 1202 Geneva, Switzerland

Taylor H. Newton, Marwan Abdellah, Grigori Chevtchenko, Eilif B. Muller, and Henry Markram

Laboratory of Neural Microcircuitry, Brain Mind Institute, EPFL, 1015 Lausanne, Switzerland Henry Markram 


\section{Contributions}

T.H.N., E.B.M., and H.M. conceptualized the study. T.H.N. designed the experiments, analyzed the data, prepared the figures, and wrote the manuscript. M.A. performed the Monte Carlo simulations of photons in brain tissue. G.C. developed the software for voxel-based calculation of the VSDI signal from membrane voltage data in consultation with T.H.N.

\section{Corresponding author}

Correspondence to Taylor H. Newton (taylor.newton@epfl.ch) 


\section{References}

Antkowiak, B. (2002). In vitro networks: cortical mechanisms of anaesthetic action. British Journal of Anaesthesia 89, 102-111.

Barreto, E., and Cressman, J.R. (2011). Ion concentration dynamics as a mechanism for neuronal bursting. J Biol Phys 37, 361-373.

Bartfeld, E., and Grinvald, A. (1992). Relationships between orientation-preference pinwheels, cytochrome oxidase blobs, and ocular-dominance columns in primate striate cortex. Proc. Natl. Acad. Sci. U.S.A. 89, 1190511909.

Bazhenov, M., Timofeev, I., Steriade, M., and Sejnowski, T.J. (2002). Model of Thalamocortical Slow-Wave Sleep Oscillations and Transitions to Activated States. J. Neurosci. 22, 8691-8704.

Berger, T., Borgdorff, A., Crochet, S., Neubauer, F.B., Lefort, S., Fauvet, B., Ferezou, I., Carleton, A., Lüscher, H.R., and Petersen, C.C.H. (2007). Combined Voltage and Calcium Epifluorescence Imaging In Vitro and In Vivo Reveals Subthreshold and Suprathreshold Dynamics of Mouse Barrel Cortex. Journal of Neurophysiology 97, 3751-3762.

Blasdel, G.G. (1992a). Differential imaging of ocular dominance and orientation selectivity in monkey striate cortex. J. Neurosci. 12, 3115-3138.

Blasdel, G.G. (1992b). Orientation selectivity, preference, and continuity in monkey striate cortex. J. Neurosci. 12, 3139-3161.

Borgdorff, A.J., Poulet, J.F.A., and Petersen, C.C.H. (2007). Facilitating Sensory Responses in Developing Mouse Somatosensory Barrel Cortex. Journal of Neurophysiology 97, 2992-3003.

Chavane, F., Sharon, D., Jancke, D., Marre, O., Frégnac, Y., and Grinvald, A. (2011). Lateral Spread of Orientation Selectivity in V1 is Controlled by Intracortical Cooperativity. Front Syst Neurosci 5, 4.

Chemla, S., and Chavane, F. (2010). Voltage-sensitive dye imaging: Technique review and models. Journal of Physiology-Paris 104, 40-50.

Civillico, E.F., and Contreras, D. (2005). Comparison of Responses to Electrical Stimulation and Whisker Deflection Using Two Different Voltage-sensitive Dyes in Mouse Barrel Cortex in Vivo. J Membrane Biol 208, 171-182.

Civillico, E.F., and Contreras, D. (2006). Integration of Evoked Responses in Supragranular Cortex Studied With Optical Recordings In Vivo. Journal of Neurophysiology 96, 336-351.

Civillico, E.F., and Contreras, D. (2012). Spatiotemporal properties of sensory responses in vivo are strongly dependent on network context. Front Syst Neurosci 6.

Colangelo, C., Shichkova, P., Keller, D., Markram, H., and Ramaswamy, S. (2019). Cellular, Synaptic and Network Effects of Acetylcholine in the Neocortex. Front Neural Circuits 13. 
Contreras, D., and Llinás, R. (2001). Voltage-Sensitive Dye Imaging of Neocortical Spatiotemporal Dynamics to Afferent Activation Frequency. J. Neurosci. 21, 9403-9413.

Contreras, D., and Steriade, M. (1996). Synchronization of low-frequency rhythms in corticothalamic networks. Neuroscience 76, 11-24.

Contreras, D., Destexhe, A., Sejnowski, T.J., and Steriade, M. (1997). Spatiotemporal Patterns of Spindle Oscillations in Cortex and Thalamus. J. Neurosci. 17, 1179-1196.

DeFelipe, J., Alonso-Nanclares, L., and Arellano, J.I. (2002). Microstructure of the neocortex: Comparative aspects. J Neurocytol 31, 299-316.

Dehghani, N., Peyrache, A., Telenczuk, B., Le Van Quyen, M., Halgren, E., Cash, S.S., Hatsopoulos, N.G., and Destexhe, A. (2016). Dynamic Balance of Excitation and Inhibition in Human and Monkey Neocortex. Scientific Reports 6, 23176.

Denève, S., and Machens, C.K. (2016). Efficient codes and balanced networks. Nature Neuroscience 19, 375-382.

Destexhe, A. (2007). High-conductance state. Scholarpedia 2, 1341.

Destexhe, A. (2010). Inhibitory “noise." Front. Cell. Neurosci. 4.

Destexhe, A., Rudolph, M., and Paré, D. (2003). The high-conductance state of neocortical neurons in vivo. Nature Reviews Neuroscience 4, 739-751.

Deuchars, J., West, D.C., and Thomson, A.M. (1994). Relationships between morphology and physiology of pyramid-pyramid single axon connections in rat neocortex in vitro. J. Physiol. (Lond.) 478 Pt 3, 423-435.

Ding, F., O'Donnell, J., Xu, Q., Kang, N., Goldman, N., and Nedergaard, M. (2016). Changes in the composition of brain interstitial ions control the sleep - wake cycle. Science 352, 550-555.

Dorn, J.D., and Ringach, D.L. (2003). Estimating Membrane Voltage Correlations From Extracellular Spike Trains. Journal of Neurophysiology 89, 2271-2278.

Eriksson, D., Tompa, T., and Roland, P.E. (2008). Non-Linear Population Firing Rates and Voltage Sensitive Dye Signals in Visual Areas 17 and 18 to Short Duration Stimuli. PLOS ONE 3, e2673.

Fehérvári, T.D., Okazaki, Y., Sawai, H., and Yagi, T. (2015). In Vivo Voltage-Sensitive Dye Study of Lateral Spreading of Cortical Activity in Mouse Primary Visual Cortex Induced by a Current Impulse. PLOS ONE 10, e0133853.

Ferezou, I., Bolea, S., and Petersen, C.C.H. (2006). Visualizing the Cortical Representation of Whisker Touch: Voltage-Sensitive Dye Imaging in Freely Moving Mice. Neuron 50, 617-629.

Ferezou, I., Haiss, F., Gentet, L.J., Aronoff, R., Weber, B., and Petersen, C.C.H. (2007). Spatiotemporal Dynamics of Cortical Sensorimotor Integration in Behaving Mice. Neuron 56, 907-923. 
Ferezou, I., Matyas, F., and Petersen, C.C.H. (2009). Imaging the Brain in Action: Real-Time Voltage- Sensitive Dye Imaging of Sensorimotor Cortex of Awake Behaving Mice. In In Vivo Optical Imaging of Brain Function, R.D. Frostig, ed. (Boca Raton (FL): CRC Press/Taylor \& Francis), p.

Fino, E., and Yuste, R. (2011). Dense Inhibitory Connectivity in Neocortex. Neuron 69, 1188-1203.

Frick, A., Feldmeyer, D., Helmstaedter, M., and Sakmann, B. (2008). Monosynaptic connections between pairs of L5A pyramidal neurons in columns of juvenile rat somatosensory cortex. Cereb. Cortex 18, 397-406.

Frostig, R.D., Lieke, E.E., Ts'o, D.Y., and Grinvald, A. (1990). Cortical functional architecture and local coupling between neuronal activity and the microcirculation revealed by in vivo high-resolution optical imaging of intrinsic signals. PNAS 87, 6082-6086.

Gil, Z., Connors, B.W., and Amitai, Y. (1999). Efficacy of Thalamocortical and Intracortical Synaptic Connections: Quanta, Innervation, and Reliability. Neuron 23, 385-397.

Gleichmann, M., and Mattson, M.P. (2010). Neuronal Calcium Homeostasis and Dysregulation. Antioxidants \& Redox Signaling 14, 1261-1273.

Gollnick, C.A., Millard, D.C., Ortiz, A.D., Bellamkonda, R.V., and Stanley, G.B. (2016). Response reliability observed with voltage-sensitive dye imaging of cortical layer 2/3: the probability of activation hypothesis. Journal of Neurophysiology 115, 2456-2469.

González-Burgos, G., Barrionuevo, G., and Lewis, D.A. (2000). Horizontal Synaptic Connections in Monkey Prefrontal Cortex: An In Vitro Electrophysiological Study. Cereb Cortex 10, 82-92.

Greenberg, D.S., Houweling, A.R., and Kerr, J.N.D. (2008). Population imaging of ongoing neuronal activity in the visual cortex of awake rats. Nature Neuroscience 11, 749-751.

Grinvald, A., and Hildesheim, R. (2004). VSDI: a new era in functional imaging of cortical dynamics. Nat Rev Neurosci 5, 874-885.

Grinvald, A., Lieke, E., Frostig, R.D., Gilbert, C.D., and Wiesel, T.N. (1986). Functional architecture of cortex revealed by optical imaging of intrinsic signals. Nature $324,361$.

Grinvald, A., Shoham, D., Shmuel, A., Glaser, D., Vanzetta, I., Shtoyerman, E., Slovin, H., Wijnbergen, C., Hildesheim, R., and Arieli, A. (1999). In-vivo Optical Imaging of Cortical Architecture and Dynamics. In Modern Techniques in Neuroscience Research, U. Windhorst, and H. Johansson, eds. (Berlin, Heidelberg: Springer Berlin Heidelberg), pp. 893-969.

Grinvald, A., Omer, D., Naaman, S., and Sharon, D. (2015). Imaging the Dynamics of Mammalian Neocortical Population Activity In-Vivo. In Membrane Potential Imaging in the Nervous System and Heart, M. Canepari, D. Zecevic, and O. Bernus, eds. (Cham: Springer International Publishing), pp. 243-271.

Grinvald, A., Omer, D.B., Sharon, D., Vanzetta, I., and Hildesheim, R. (2016). Voltage-Sensitive Dye Imaging of Neocortical Activity. Cold Spring Harb Protoc 2016, pdb.top089367.

Henn, F.A., Haljama“e, H., and Hamberger, A. (1972). Glial cell function: Active control of extracellular K+ concentration. Brain Research 43, 437-443. 
Hines, M.L., and Carnevale, N.T. (1997). The NEURON Simulation Environment. Neural Computation 9, 11791209.

Hughes, S.W., Cope, D.W., Blethyn, K.L., and Crunelli, V. (2002). Cellular Mechanisms of the Slow (<1 Hz) Oscillation in Thalamocortical Neurons In Vitro. Neuron 33, 947-958.

Huys, R., Jirsa, V.K., Darokhan, Z., Valentiniene, S., and Roland, P.E. (2016). Visually Evoked Spiking Evolves While Spontaneous Ongoing Dynamics Persist. Front Syst Neurosci 9.

Isaacson, J.S., and Scanziani, M. (2011). How Inhibition Shapes Cortical Activity. Neuron 72, 231-243.

Jin, W., Zhang, R.-J., and Wu, J. (2002). Voltage-sensitive dye imaging of population neuronal activity in cortical tissue. Journal of Neuroscience Methods 115, 13-27.

Kapfer, C., Glickfeld, L.L., Atallah, B.V., and Scanziani, M. (2007). Supralinear increase of recurrent inhibition during sparse activity in the somatosensory cortex. Nat. Neurosci. 10, 743-753.

Karnani, M.M., Agetsuma, M., and Yuste, R. (2014). A blanket of inhibition: functional inferences from dense inhibitory connectivity. Current Opinion in Neurobiology 26, 96-102.

Kawaguchi, Y. (2017). Pyramidal Cell Subtypes and Their Synaptic Connections in Layer 5 of Rat Frontal Cortex. Cereb Cortex 27, 5755-5771.

Kelly, J.P., and Essen, D.C.V. (1974). Cell structure and function in the visual cortex of the cat. The Journal of Physiology 238, 515-547.

Kleinfeld, D., and Delaney, K. r. (1996). Distributed representation of vibrissa movement in the upper layers of somatosensory cortex revealed with voltage-sensitive dyes. J. Comp. Neurol. 375, 89-108.

Kraio, R.P., and Nicholson, C. (1978). Extracellular ionic variations during spreading depression. Neuroscience 3, 1045-1059.

Kreuzer, M., Hentschke, H., Antkowiak, B., Schwarz, C., Kochs, E.F., and Schneider, G. (2010). Cross-approximate entropy of cortical local field potentials quantifies effects of anesthesia - a pilot study in rats. BMC Neuroscience $11,122$.

Kuhn, A., Aertsen, A., and Rotter, S. (2004). Neuronal Integration of Synaptic Input in the Fluctuation-Driven Regime. J. Neurosci. 24, 2345-2356.

Kyriakatos, A., Sadashivaiah, V., Zhang, Y., Motta, A., Auffret, M., and Petersen, C.C.H. (2017). Voltage-sensitive dye imaging of mouse neocortex during a whisker detection task. Neurophotonics 4.

Lippert, M., Takagaki, K., Xu, W., Huang, X., and Wu, J.-Y. (2007). Methods for voltage-sensitive dye imaging of rat cortical activity with high signal-to-noise ratio. J Neurophysiol 98, 502-512.

Lustig, B.R., Friedman, R.M., Winberry, J.E., Ebner, F.F., and Roe, A.W. (2013). Voltage-sensitive dye imaging reveals shifting spatiotemporal spread of whisker-induced activity in rat barrel cortex. Journal of Neurophysiology 109, 2382-2392. 
Markram, H., Lübke, J., Frotscher, M., Roth, A., and Sakmann, B. (1997). Physiology and anatomy of synaptic connections between thick tufted pyramidal neurones in the developing rat neocortex. J. Physiol. (Lond.) 500 ( Pt 2), 409-440.

Markram, H., Muller, E., Ramaswamy, S., Reimann, M.W., Abdellah, M., Sanchez, C.A., Ailamaki, A., AlonsoNanclares, L., Antille, N., Arsever, S., et al. (2015). Reconstruction and Simulation of Neocortical Microcircuitry. Cell 163, 456-492.

McDonald, C.T., and Burkhalter, A. (1993). Organization of long-range inhibitory connections with rat visual cortex. J. Neurosci. 13, 768-781.

Mena-Segovia, J., Sims, H.M., Magill, P.J., and Bolam, J.P. (2008). Cholinergic brainstem neurons modulate cortical gamma activity during slow oscillations. The Journal of Physiology 586, 2947-2960.

Mesradi, M., Genoux, A., Cuplov, V., Abi Haidar, D., Jan, S., Buvat, I., and Pain, F. (2013). Experimental and analytical comparative study of optical coefficient of fresh and frozen rat tissues. J Biomed Opt 18, 117010.

Muller, E., Buesing, L., Schemmel, J., and Meier, K. (2007). Spike-frequency adapting neural ensembles: beyond mean adaptation and renewal theories. Neural Comput 19, 2958-3010.

Murphy, M., Bruno, M.-A., Riedner, B.A., Boveroux, P., Noirhomme, Q., Landsness, E.C., Brichant, J.-F., Phillips, C., Massimini, M., Laureys, S., et al. (2011). Propofol Anesthesia and Sleep: A High-Density EEG Study. Sleep 34, 283-291.

Orbach, H.S., Cohen, L.B., and Grinvald, A. (1985). Optical mapping of electrical activity in rat somatosensory and visual cortex. J. Neurosci. 5, 1886-1895.

Petersen, C.C.H. (2007). The Functional Organization of the Barrel Cortex. Neuron 56, 339-355.

Petersen, C.C.H., and Sakmann, B. (2001). Functionally Independent Columns of Rat Somatosensory Barrel Cortex Revealed with Voltage-Sensitive Dye Imaging. J. Neurosci. 21, 8435-8446.

Petersen, C.C.H., Hahn, T.T.G., Mehta, M., Grinvald, A., and Sakmann, B. (2003a). Interaction of sensory responses with spontaneous depolarization in layer 2/3 barrel cortex. PNAS 100, 13638-13643.

Petersen, C.C.H., Grinvald, A., and Sakmann, B. (2003b). Spatiotemporal Dynamics of Sensory Responses in Layer $2 / 3$ of Rat Barrel Cortex Measured In Vivo by Voltage-Sensitive Dye Imaging Combined with Whole-Cell Voltage Recordings and Neuron Reconstructions. J. Neurosci. 23, 1298-1309.

Pharr, M., Jakob, W., and Humphreys, G. (2016). Physically Based Rendering: From Theory to Implementation (San Francisco, CA, USA: Morgan Kaufmann Publishers Inc.).

Rasmussen, R., Jensen, M.H., and Heltberg, M.L. (2017). Chaotic Dynamics Mediate Brain State Transitions, Driven by Changes in Extracellular lon Concentrations. Cels 5, 591-603.e4.

Ratzlaff, E.H., and Grinvald, A. (1991). A tandem-lens epifluorescence macroscope: Hundred-fold brightness advantage for wide-field imaging. Journal of Neuroscience Methods 36, 127-137. 
Reimann, M.W., King, J.G., Muller, E.B., Ramaswamy, S., and Markram, H. (2015). An algorithm to predict the connectome of neural microcircuits. Front Comput Neurosci 9.

Reimann, M.W., Gevaert, M., Shi, Y., Lu, H., Markram, H., and Muller, E. (2019). A null model of the mouse whole-neocortex micro-connectome. Nat Commun 10, 1-16.

Roland, P.E. (2017). Space-Time Dynamics of Membrane Currents Evolve to Shape Excitation, Spiking, and Inhibition in the Cortex at Small and Large Scales. Neuron 94, 934-942.

Roland, P.E., Hanazawa, A., Undeman, C., Eriksson, D., Tompa, T., Nakamura, H., Valentiniene, S., and Ahmed, B. (2006). Cortical feedback depolarization waves: A mechanism of top-down influence on early visual areas. PNAS $103,12586-12591$.

Rudolph, M., Piwkowska, Z., Badoual, M., Bal, T., and Destexhe, A. (2004). A Method to Estimate Synaptic Conductances From Membrane Potential Fluctuations. Journal of Neurophysiology 91, 2884-2896.

Rudolph, M., Pelletier, J.G., Paré, D., and Destexhe, A. (2005). Characterization of Synaptic Conductances and Integrative Properties During Electrically Induced EEG-Activated States in Neocortical Neurons In Vivo. Journal of Neurophysiology 94, 2805-2821.

Schlaggar, B.L., and O'Leary, D.D.M. (1994). Early development of the somatotopic map and barrel patterning in rat somatosensory cortex. Journal of Comparative Neurology 346, 80-96.

Schummers, J., Yu, H., and Sur, M. (2008). Tuned Responses of Astrocytes and Their Influence on Hemodynamic Signals in the Visual Cortex. Science 320, 1638-1643.

Sengupta, B., Laughlin, S.B., and Niven, J.E. (2013). Balanced Excitatory and Inhibitory Synaptic Currents Promote Efficient Coding and Metabolic Efficiency. PLOS Computational Biology 9, e1003263.

Sharon, D., and Grinvald, A. (2002). Dynamics and Constancy in Cortical Spatiotemporal Patterns of Orientation Processing. Science 295, 512-515.

Shoham, D., Glaser, D.E., Arieli, A., Kenet, T., Wijnbergen, C., Toledo, Y., Hildesheim, R., and Grinvald, A. (1999). Imaging Cortical Dynamics at High Spatial and Temporal Resolution with Novel Blue Voltage-Sensitive Dyes. Neuron 24, 791-802.

Silberberg, G., and Markram, H. (2007). Disynaptic inhibition between neocortical pyramidal cells mediated by Martinotti cells. Neuron 53, 735-746.

Silver, R.A., Lubke, J., Sakmann, B., and Feldmeyer, D. (2003). High-probability uniquantal transmission at excitatory synapses in barrel cortex. Science 302, 1981-1984.

Simons, D.J., Durham, D., and Woolsey, T.A. (1984). Functional Organization of Mouse and Rat Sml Barrel Cortex following Vibrissal Damage on Different Postnatal Days. Somatosensory Research 1, 207-245.

Steriade, M. (2000). Corticothalamic resonance, states of vigilance and mentation. Neuroscience 101, $243-276$.

Steriade, M., McCormick, D.A., and Sejnowski, T.J. (1993). Thalamocortical oscillations in the sleeping and aroused brain. Science 262, 679-685. 
Timofeev, I., Grenier, F., Bazhenov, M., Sejnowski, T.J., and Steriade, M. (2000). Origin of Slow Cortical Oscillations in Deafferented Cortical Slabs. Cereb Cortex 10, 1185-1199.

Tomioka, R., Okamoto, K., Furuta, T., Fujiyama, F., Iwasato, T., Yanagawa, Y., Obata, K., Kaneko, T., and Tamamaki, N. (2005). Demonstration of long-range GABAergic connections distributed throughout the mouse neocortex. European Journal of Neuroscience 21, 1587-1600.

Tsau, Y., Wenner, P., O’Donovan, M.J., Cohen, L.B., Loew, L.M., and Wuskell, J.P. (1996). Dye screening and signal-to-noise ratio for retrogradely transported voltage-sensitive dyes. Journal of Neuroscience Methods 70 , 121-129.

Ts'o, D.Y., Frostig, R.D., Lieke, E.E., and Grinvald, A. (1990). Functional organization of primate visual cortex revealed by high resolution optical imaging. Science $249,417-420$.

Wang, Y., Gupta, A., Toledo-Rodriguez, M., Wu, C.Z., and Markram, H. (2002). Anatomical, physiological, molecular and circuit properties of nest basket cells in the developing somatosensory cortex. Cereb. Cortex 12, 395-410.

Zerlaut, Y., Chemla, S., Chavane, F., and Destexhe, A. (2018). Modeling mesoscopic cortical dynamics using a mean-field model of conductance-based networks of adaptive exponential integrate-and-fire neurons. J Comput Neurosci 44, 45-61.

Zhou, S., and Yu, Y. (2018). Synaptic E-I Balance Underlies Efficient Neural Coding. Front. Neurosci. 12.

Zhou, W.-L., Yan, P., Wuskell, J.P., Loew, L.M., and Antic, S.D. (2007). Intracellular long-wavelength voltagesensitive dyes for studying the dynamics of action potentials in axons and thin dendrites. Journal of Neuroscience Methods 164, 225-239. 